\title{
The Effect of Nondeterministic Parameters on Shock-Associated Noise Prediction Modeling
}

Milo D. Dahl

Glenn Research Center, Cleveland, Ohio

Abbas Khavaran

ASRC Aerospace Corporation, Cleveland, Ohio 


\section{NASA STI Program . . . in Profile}

Since its founding, NASA has been dedicated to the advancement of aeronautics and space science. The NASA Scientific and Technical Information (STI) program plays a key part in helping NASA maintain this important role.

The NASA STI Program operates under the auspices of the Agency Chief Information Officer. It collects, organizes, provides for archiving, and disseminates NASA's STI. The NASA STI program provides access to the NASA Aeronautics and Space Database and its public interface, the NASA Technical Reports Server, thus providing one of the largest collections of aeronautical and space science STI in the world. Results are published in both non-NASA channels and by NASA in the NASA STI Report Series, which includes the following report types:

- TECHNICAL PUBLICATION. Reports of completed research or a major significant phase of research that present the results of NASA programs and include extensive data or theoretical analysis. Includes compilations of significant scientific and technical data and information deemed to be of continuing reference value. NASA counterpart of peer-reviewed formal professional papers but has less stringent limitations on manuscript length and extent of graphic presentations.

- TECHNICAL MEMORANDUM. Scientific and technical findings that are preliminary or of specialized interest, e.g., quick release reports, working papers, and bibliographies that contain minimal annotation. Does not contain extensive analysis.

- CONTRACTOR REPORT. Scientific and technical findings by NASA-sponsored contractors and grantees.
- CONFERENCE PUBLICATION. Collected papers from scientific and technical conferences, symposia, seminars, or other meetings sponsored or cosponsored by NASA.

- SPECIAL PUBLICATION. Scientific, technical, or historical information from NASA programs, projects, and missions, often concerned with subjects having substantial public interest.

- TECHNICAL TRANSLATION. Englishlanguage translations of foreign scientific and technical material pertinent to NASA's mission.

Specialized services also include creating custom thesauri, building customized databases, organizing and publishing research results.

For more information about the NASA STI program, see the following:

- Access the NASA STI program home page at http://www.sti.nasa.gov

- E-mail your question via the Internet to help@ sti.nasa.gov

- Fax your question to the NASA STI Help Desk at 443-757-5803

- Telephone the NASA STI Help Desk at 443-757-5802

- Write to: NASA Center for AeroSpace Information (CASI) 7115 Standard Drive Hanover, MD 21076-1320 


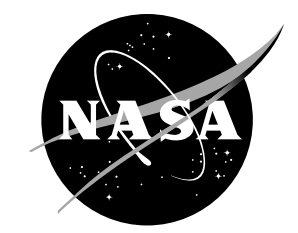

\section{The Effect of Nondeterministic Parameters on Shock-Associated Noise Prediction Modeling}

Milo D. Dahl

Glenn Research Center, Cleveland, Ohio

Abbas Khavaran

ASRC Aerospace Corporation, Cleveland, Ohio

Prepared for the

16th Aeroacoustics Conference

cosponsored by the American Institute of Aeronautics and Astronautics and the Confederation of European Aerospace Societies

Stockholm, Sweden, June 7-9, 2010

National Aeronautics and

Space Administration

Glenn Research Center

Cleveland, Ohio 44135 


\section{Acknowledgments}

The NASA Supersonics Project of the Fundamental Aeronautics Program supported this work.

This work was sponsored by the Fundamental Aeronautics Program at the NASA Glenn Research Center.

Level of Review: This material has been technically reviewed by technical management.

Available from

NASA Center for Aerospace Information 7115 Standard Drive

Hanover, MD 21076-1320
National Technical Information Service 5301 Shawnee Road Alexandria, VA 22312

Available electronically at http://gltrs.grc.nasa.gov 


\title{
The Effect of Nondeterministic Parameters on Shock-Associated Noise Prediction Modeling
}

\author{
Milo D. Dahl \\ National Aeronautics and Space Administration \\ Glenn Research Center \\ Cleveland, Ohio 44135 \\ Abbas Khavaran \\ ASRC Aerospace Corporation \\ Cleveland, Ohio 44135
}

\begin{abstract}
Engineering applications for aircraft noise prediction contain models for physical phenomenon that enable solutions to be computed quickly. These models contain parameters that have an uncertainty not accounted for in the solution. To include uncertainty in the solution, nondeterministic computational methods are applied. Using prediction models for supersonic jet broadband shock-associated noise, fixed model parameters are replaced by probability distributions to illustrate one of these methods. The results show the impact of using nondeterministic parameters both on estimating the model output uncertainty and on the model spectral level prediction. In addition, a global sensitivity analysis is used to determine the influence of the model parameters on the output, and to identify the parameters with the least influence on model output.
\end{abstract}

\section{Nomenclature}

$\begin{array}{ll}A & \text { Area } \\ B & \text { Intercept parameter, see equation (3) } \\ D & \text { Diameter } \\ E & \text { Amplitude power exponent parameter, see equation (6) } \\ \mathrm{J}_{0} & \text { Bessel function of the first kind, order zero } \\ \mathrm{J}_{1} & \text { Bessel function of the first kind, order one } \\ L & \text { Gaussian source half-width function, see equation }(7) \\ M & \text { Mach number } \\ N & \text { Total number of random samples } \\ P & \text { Pressure } \\ R & \text { Radial distance } \\ S_{T i} & \text { Total effects sensitivity measure, see equation }(1) \\ S t & \text { Strouhal number } \\ T & \text { Temperature } \\ U & \text { Velocity } \\ X_{i} & i \text {-th parameter in vector } \boldsymbol{X} \\ Y & \text { output quantity from model with parameters } \boldsymbol{X} \\ \boldsymbol{X} & \text { Vector of parameters } \\ \boldsymbol{X}^{*} & \boldsymbol{X} \text { fixed at a set of values } \\ \mathrm{E}\{\} & \text { Expected value of the enclosed quantity } \\ \text { Var }() & \text { Variance of the enclosed quantity } \\ \bar{A}^{2} & \text { Shock cell strength amplitude function, see equation }(7) \\ \bar{c} & \text { Amplitude factor, see equation }(7) \\ c & \text { Speed of sound } \\ & \end{array}$




$\begin{array}{ll}f & \text { Frequency } \\ j_{0, \nu} & \text { } \text {-th zero of a zeroth order Bessel function } \\ m & \text { Shock power factor } \\ \left\langle p^{2}\right\rangle & \text { Mean-square acoustic pressure } \\ x & \text { Independent regression variable } \\ y & \text { Dependent regression variable } \\ z & \text { Linear regression residual error, see equation (5) } \\ \text { Subscripts } & \text { Ambient conditions } \\ \infty & \text { integer index, maximum value } N_{\nu} \\ \nu, N_{\nu} & \text { All parameters in vector not including the } i \text {-th parameter } \\ \sim i & \text { Convective velocity or Mach number } \\ c & \text { Design conditions } \\ d & \text { Nozzle exit conditions } \\ e & \text { integer index, maximum value } I \\ i, I & \text { Fully expanded jet conditions } \\ j & \text { integer index, maximum value } K \\ k, K & \text { Randomly selected variable } \\ r & \text { Shock-associated noise related } \\ s h & \text { Total conditions } \\ t & \\ S y m b o l s & \text { Shock parameter } \\ \beta & \text { Chi-squared distribution with } n \text {-degrees of freedom } \\ \chi_{n}^{2} & \text { Nonlinear regression residual error, see equation }(6) \\ \epsilon & \text { Ratio of specific heats } \\ \gamma & \text { Density with one subscript } \\ \rho_{1} & \text { Correlation coefficient with two subscripts } \\ \rho_{12} & \text { Standard deviation } \\ \sigma & \text { Inlet directivity angle } \\ N\left(\mu, \sigma^{2}\right) & \text { Normal distribution with mean } \mu \text { and variance } \sigma^{2} \\ & \end{array}$

\section{Introduction}

The development of tools to computationally predict the sound generated by fluid flow or when fluid interacts with a surface continues to be an important part of the research effort for developing future environmentally friendly aircraft. For aircraft propulsion system related jet noise, a wide range of computer-based schemes are included in a hierarchy of these noise prediction tools from numerically intensive, extremely long running, direct simulations based on solving first principle equations to near instantly computed, empirical methods using experimental databases. The numerically intensive computations may simulate all the proper physics for jet noise predictions, but running them is so costly that they currently have little practical usage. At the other extreme, the empirical models are limited in their ability to predict jet noise beyond a relatively small set of geometries and operating conditions with which they were developed. In between these two extremes are found methods that use some combination of numerical techniques and physics-based models in an attempt to increase the practical ability to predict jet noise over a wider range of jet operating conditions and nozzle geometries. Among these are included tools to compute the unsteady fluid flow using various types of filtering and turbulence modeling schemes to obtain a noise source field that is used to compute the radiated noise field, and tools that use acoustic analogy source models based on mean fluid turbulent statistical values to compute a solution for the radiated noise field. Even though these latter numerical methods still include some level of modeling that reduces total computation time, the time required to compute the unsteady or the steady mean flow can inhibit the number of cases run for a design study.

For engineering applications in aircraft noise prediction at the component and especially at the system level, extensive modeling of the physical phenomena is required to obtain many solutions for design, development, and parametric studies. Analytical development and intuition are used to develop models that describe physical phenomena. In the end, these models will contain parameters that must be determined 
by optimization and calibration. These parameters are found by using numerical simulation and/or experimental data. Regression is often used to set these parameters. This deterministic approach may provide reasonable solutions, but usually within limited conditions. However, the determination or the calibration of these parameters is often inexact especially when using experimental data that contains uncertainty, that may be insufficient for good statistics, or that may be of poor quality. Thus there is a question if the best-fit parameters are truly the optimal parameters for the model. It may be more appropriate to include the parameter uncertainty in the model ${ }^{1}$ and/or to compute the solution using nondeterministic methods. ${ }^{2}$ In the present paper, we consider the introduction of nondeterministic parameters into models for the prediction of broadband, shock-associated noise from supersonic jets. This was done as part of a broader study and assessment of jet noise prediction models both as a method of determining the quality of the prediction and as a method of providing better prediction capability. Nondeterministic approaches are shown to impact the predicted results and can be used to estimate the output uncertainty and to determine the influence of the model parameters on the output.

A brief background for this paper is given in the next section. A previous assessment study of jet noise prediction models, including models for broadband shock-associated noise, determined the uncertainty in the output from the prediction models. The shock-associated noise prediction models were easily modified to apply the method of computing with nondeterministic parameters. This is followed by a section describing some of the details of the prediction models. One model relies on a two-parameter regression analysis of experimental data to provide a scheme to predict noise amplitude as a function of jet operating conditions and directivity angle. The regression analysis provides the statistical quantities to define the distribution for the uncertain parameters. The other model has a large number of parameters with unknown distributions. The results are then presented in three parts. The first discusses the effects of the input operating condition uncertainty on model output uncertainty. Next, the results for the model using the regression analysis is presented. The main issue is which type of regression is appropriate. The last part presents results for the many parameter model. This part focuses on the choice of distribution for the uncertain parameters and the determination of the influence of the parameters on the output. This helps identify parameters that most affect the output uncertainty.

\section{Background and Methodology}

Bridges et al. ${ }^{3}$ assessed the capability to predict jet noise and showed results from empirical and acoustic analogy based tools compared to measured data over a wide range of operating conditions. A general conclusion was that empirical tools could make overall sound pressure level predictions to within $\pm 6 \mathrm{~dB}$ of measured values for a broad range of nozzle shapes and operating conditions when averaged over all directivity angles. For simple axisymmetric nozzles, the predictions improved to agree within $\pm 1.2 \mathrm{~dB}$. In comparison, an acoustic analogy tool was only applicable to a limited range of conditions consisting of axisymmetric, cold subsonic jets. The accuracy for these comparisons were also within $\pm 1.2 \mathrm{~dB}$. That study showed some of the issues related to how the results from different jet noise prediction models compare to the same set of observations and how to judge the quality of those predictions. Khavaran \& Bridges ${ }^{4}$ further analyzed the measured jet noise data at supersonic conditions, separating mixing noise and shockassociated noise spectral components from the total jet noise spectrum, to provide better data for jet noise model predictions and improvements. The results of that component separation allow us to focus this study on shock-associated noise prediction capabilities for jets from convergent nozzles. To illustrate the results from different models, example spectra that compare predictions for shock-associated noise with the shock-associated noise component of measured data are shown in Figure 1. The plots show power spectral density as a function of the Strouhal number for under-expanded jets with a fully expanded Mach number of about 1.5. There are two jet temperatures represented at two directivity angles from the inlet of 50 and 90 degrees. Three predictions are shown from the following models: The analytically based shock-associated noise prediction model from Tam, ${ }^{5}$ the intensity scaling prediction model based on the work of Khavaran \& Bridges $^{6}$ and further detailed in Appendix A of this paper (labeled $s J e t$ ), and the Reynolds-average-NavierStokes-based (RANS) model of Morris \& Miller. ${ }^{7}$ Three different models attempting to predict the same experimental results.

The shock-associated noise models under consideration here were originally developed to make deterministic, one-time calculations using parameters found through least-squares fitting, averaging, point matching, or other methods to calibrate computed predictions using experimental data. These fixed parameters in 
reality have an uncertainty associated with them that is ignored in the basic usage of these prediction models. To incorporate the uncertainty, knowledge about the quantities that describe the uncertainty must be available and/or the distribution of the uncertain parameter must be known or estimated. This information may be unknown or hard to obtain. In the case of the sJet code, the uncertain parameters are well described in terms of their probability distributions and the moments associated with those distributions. Thus, it is readily possible to replace fixed parameters with their nondeterministic distributions. Once this is done, a method must be applied to perform the probabilistic analysis. One approach is to perform Monte Carlo sampling using the original prediction model. Depending on the scale of the problem, the number of computations may become quite large. Latin hypercube sampling helps reduce the number of sampling computations required to achieve convergence for good statistics. ${ }^{8}$ Alternatives are to replace the original computational model with an analytical model that is more computationally efficient. Such methods include first and second order reliability methods, advanced mean value methods, and the response surface method. ${ }^{9}$ The analytical model may be faster to compute, but it is approximate and contains inaccuracies. The two models to be used here, the sJet and Tam models, are already approximate and are easily amenable to replacing fixed parameters with nondeterministic distributions. The RANS-based model was not available for this study. Thus, the Monte Carlo approach was chosen. A software tool that easily performs the Monte Carlo calculations using the external jet noise prediction codes is called DAKOTA. ${ }^{8}$ This program can be set up to perform the following:

1. Generate random inputs from predefined distributions.

2. Execute the prediction model code using the random inputs.

3. Perform statistical analysis on the outputs from the model code.

In addition to this Monte Carlo calculation, the DAKOTA code can perform sensitivity analyses of the parameters to determine their influence on the output, optimization studies to find the best parameters, and other related computations.

Sensitivity analysis is often used in conjunction with uncertainty analysis. While uncertainty analysis attempts to quantify the level of model output uncertainty given a set of model parameter uncertainties, sensitivity analysis is concerned with attempting to determine how much influence a particular parameter has on the output or output uncertainty and then rank the parameters in degrees of importance. This allows parameters of lower importance to be fixed and more attention and resources can be focused on parameters of higher importance. One sensitivity analysis method supported by the DAKOTA code is called variance-based decomposition. Let the parameters be denoted by the vector $\boldsymbol{X}$. If a particular parameter $X_{i}$ was absolutely non-influential, then the variance of the output $Y$ due to changes in $X_{i}$ when all the other parameters in $\boldsymbol{X}$ are fixed, denoted as $\boldsymbol{X}_{\sim i}^{*}$, must be zero. We compute the conditional variance $\operatorname{Var}_{X_{i}}\left(Y \mid \boldsymbol{X}_{\sim i}=\boldsymbol{X}_{\sim i}^{*}\right)$ where the subscript $X_{i}$ indicates the one parameter in $\boldsymbol{X}$ that is being randomly sampled while all the other parameters are held fixed. The conditional variance must be zero for a second set of $\boldsymbol{X}_{\sim i}^{*}$, a third set of $\boldsymbol{X}_{\sim i}^{*}$, and so forth. Averaging these conditional variances over the set $\boldsymbol{X}_{\sim i}$ will not change this result, hence the average will be zero, also. Comparing the average to the total variance of the output $Y$, we obtain the total effect sensitivity measure $S_{T i}$ for the parameter $X_{i}$,

$$
S_{T i}=\frac{\mathrm{E}_{\boldsymbol{X}_{\sim i}}\left\{\operatorname{Var}_{X_{i}}\left(Y \mid \boldsymbol{X}_{\sim i}\right)\right\}}{\operatorname{Var}(Y)},
$$

where $S_{T i}$ includes both a measure of the direct effect of $X_{i}$ and a measure of the interaction effects of $X_{i}$ with all the other $\boldsymbol{X}_{\sim i}$ on the output variance. In actual calculation, $S_{T i}$ will most likely be nonzero; hence, a small value for $S_{T i}$ represents an $X_{i}$ parameter with less influence on the output than a different $X_{i}$ parameter with a larger $S_{T i}$ value. Since the computation of the total effect sensitivity measure involves varying all the parameters simultaneously, it is considered to be a global measure in contrast to a local measure that involves varying only one parameter at a time. The details of variance-based decomposition and its implementation to obtain sensitivity measures are found in Saltelli et al. ${ }^{10,11}$ and the references therein.

\section{Models and Data}

This section introduces the two jet broadband shock-associated noise prediction models. The development approach for these two models were entirely different. The sJet code was developed from a study of the data, 
particularly far-field spectral densities at various directivity angles. The Tam model was developed from derivations of the equations of motion. Only after this development were simplifications and adjustments made when results were compared to measured data. The section concludes with the experimental conditions for the data used for comparison with predictions in this paper.

\section{III.A. sJet Model}

In the jet noise prediction modeling approach proposed by Khavaran \& Bridges, ${ }^{6}$ scaling laws are used to derive relationships between fundamental jet parameters and the total radiated power computed by integrating over the appropriate power spectral density. To enable this process for imperfectly expanded supersonic jets, the radiated broadband noise was separated into distinct spectral components associated with mixing noise and shock-associated noise. Here, we are only concerned with the model for shockassociated noise. The total shock-noise intensity scales with the shock parameter $\beta$, a measure of the shock strength in the flow given by the amount to which the fully expanded Mach number deviates from the nozzle design Mach number. In general, this may be expressed as

$$
\left\langle p^{2}\right\rangle_{s h} \propto \beta^{m} \quad \text { where } \beta^{2}=\left|M_{j}^{2}-M_{d}^{2}\right| .
$$

Based on experimental measurements, the shock power factor $m$ varies with the noise radiation directivity angle and the jet temperature.

Following Khavaran \& Bridges, ${ }^{6}$ a linear regression is applied in the form

$$
y_{i}=m(\theta, T) x_{i}+B(\theta, T)
$$

where the dependent variable $y_{i}$ and the independent variable $x_{i}$ are given by

$$
y_{i}=\mathrm{OASPL}_{s h}(\theta, T) \quad \text { and } \quad x_{i}=10 \log \beta_{i}, \quad i=1,2, \ldots, I .
$$

The overall sound pressure level (OASPL) is for the shock-associated noise computed from the shockassociated noise spectral component of the total radiated noise over the Strouhal number range $0.1 \leq S t \leq 10$ where $S t=f D_{e} / U_{j}$. The intercept parameter $B$ is a measure of the shock noise overall level in decibels when the shock parameter $\beta$ equals one. The results of the linear regression are the mean values $\bar{m}$ and $\bar{B}$, the standard deviations $\sigma_{m}$ and $\sigma_{B}$, and the correlation coefficient $\rho_{m B}$ between the $m$ and $B$ parameters. Once $m$ and $B$ are determined from multiple linear regressions covering a range of inlet directivity angles $\theta$ and total temperatures $T_{t}$, the shock-associated noise levels are now set in general and it remains to compute the spectral shape. See Appendix A for the detailed equations.

The linear regression model given in equation (3) is based on the general model regression equation

$$
y_{i}=m x_{i}+B+z_{i}
$$

where the $z_{i}$ 's are assumed to be independent, normally-distributed, random values with zero mean and equal variances representing the random or residual error in the linear regression. ${ }^{12}$ This assumption is most likely not strictly maintained in the above linear regression. Equation (3) is a logarithmic transformation of equation (2). While this creates a linear equation from a nonlinear equation, the errors are also transformed and may violate the assumption for $z_{i}$ in equation (5). ${ }^{13}$ In the original formulation, $\left\langle p^{2}\right\rangle_{s h}$ is an overall power level computed by adding together the power levels in each frequency bin of the shock-associated noise power spectral density (PSD). Each power level at each frequency in the PSD has a chi-squared distribution $\chi_{n}^{2}$ with $n$-degrees of freedom after spectral averaging. ${ }^{14}$ A summation over $K$ frequencies in a power spectral density with each power level having a $\chi_{n}^{2}$ distribution results in a total power level with a chi-squared distribution $\chi_{K \times n}^{2}$ with $(K \times n)$-degrees of freedom. A nonlinear regression equation for the total power level can then be written as

$$
y_{i}=10^{E(\theta, T)} \beta_{i}^{m(\theta, T)}+\epsilon_{i}
$$

where both $y_{i}=\left\langle p^{2}\right\rangle_{s h}(\theta, T)$ and $\epsilon_{i}$, the residual error, have in general a $\chi_{K \times n}^{2}$-distribution. Using $10^{E}$ is a recognition that $\left\langle p^{2}\right\rangle_{s h}$ has a potential range covering many orders of magnitude. It can be shown that when weighting is used and the chi-squared distribution has a large number of degrees of freedom, the nonlinear regression formulation of equation (6) is appropriate for the given data and that the residual errors more closely have the desired normally-distributed properties. See Appendix B for details. 


\section{III.B. Tam Model}

The broadband shock-associated noise model of $\operatorname{Tam}^{5,15}$ is based on the theory that large-scale turbulent structures generated in the supersonic jet mixing layer interact with the periodic-like shock cell structure that exists in imperfectly expanded jets. This interaction causes pressure perturbations that radiate as sound. The shock cell structure is modeled as a wave guide containing a series of modes and the large structures are modeled as a sum of instability waves each with their own amplitude envelope and phase speed characteristics. These characteristics affect the amplitude and directivity of the radiated noise. The model is applicable to both under-expanded and over-expanded jets, but in this paper, it is applied only to under-expanded jets issuing from convergent nozzles.

Following extensive algebraic manipulations and modeling approximations, Tam $^{5}$ derived an equation for the far field mean-square acoustic pressure

$$
\left\langle p^{2}\right\rangle_{k}=\frac{\rho_{j}}{\rho_{\infty}}\left[1+\frac{\gamma-1}{2} M_{j}^{2}\right]^{-1} \frac{\bar{c}\left(c_{b 1}\right) L^{2}\left(h_{s 1}, x_{c 1}, x_{c 2}\right) A_{j} \bar{A}^{2}\left(a_{b 1}, a_{b 2}\right) \rho_{\infty}^{2} c_{\infty}^{2} U_{j}^{2}}{R^{2} S t_{k} f_{k}} \sum_{\nu=1}^{N_{\nu}} \frac{1}{j_{0, \nu}^{2} J_{1}^{2}\left(j_{0, \nu}\right)} \exp \left[\mathcal{F}_{\nu}\right]
$$

and

$$
\mathcal{F}_{\nu}=-\left(\frac{f_{\nu}\left(v_{c 1}, v_{c 2}, w_{11}, w_{12}, w_{21}, w_{\nu 1}\right)}{f_{k}}-1\right)^{2} \frac{L^{2}\left(h_{s 1}, x_{c 1}, x_{c 2}\right)}{2 \ln 2}\left(\frac{U_{j}}{U_{c}\left(v_{c 1}, v_{c 2}\right)}\right)^{2}\left(1+M_{c}\left(v_{c 1}, v_{c 2}\right) \cos \theta\right)^{2}
$$

where $\rho_{j}, U_{j}, M_{j}$, and $A_{j}$ are the density, velocity, Mach number, and cross-sectional area, respectively, of the jet at the fully expanded condition, $\rho_{\infty}$ is the ambient density, $c_{\infty}$ is the ambient speed of sound, $R$ is the radial observer distance, $\theta$ is the inlet directivity angle, $f_{k}$ is the $k$-th frequency in a spectrum, $S t_{k}$ is the Strouhal number $f_{k} D_{j} / U_{j}, D_{j}$ is the diameter corresponding to $A_{j}$, and $f_{\nu}$ is the peak frequency corresponding to the mode number $\nu$. The denominator factor $S t_{k}$ in equation (7) is fixed at 0.35 when $S t_{k}<0.35$. In the summation of waveguide modes, $\mathrm{J}_{1}$ is a first order Bessel function and $j_{0, \nu}$ is the $\nu$-th zero of the zeroth order Bessel function $\mathrm{J}_{0}$. The functional arguments with two subscripts represent the twelve parameters in equation (7) that were derived by fitting equations to experimental or calculated data. Table 1 defines these parameters and the detailed equations are given in Appendix C.

\begin{tabular}{|c|c|c|}
\hline Parameter & Contributes to & Model or Mean Value \\
\hline$a_{b 1}$ & amplitude & $3\left(M_{j} / M_{d} \geq 1\right), 6\left(M_{j} / M_{d}<1\right)$ \\
$a_{b 2}$ & amplitude & $3\left(M_{j} / M_{d} \geq 1\right), 5\left(M_{j} / M_{d}<1\right)$ \\
$w_{11}$ & 1st waveguide mode wave number & 1.596 \\
$w_{12}$ & 1st waveguide mode wave number & 0.1773 \\
$w_{21}$ & 2nd waveguide mode wave number & 1.265 \\
$w_{\nu 1}$ & $\nu$-th waveguide mode wave number & 1.1 \\
$c_{b 1}$ & amplitude & $2.65 \times 10^{-4}$ \\
$h_{s 1}$ & source half-width length scale & 3.3 \\
$x_{c 1}$ & potential core length & 4.3 \\
$x_{c 2}$ & potential core length & 1.2 \\
$v_{c 1}$ & convective velocity & 0.7 \\
$v_{c 2}$ & convective velocity & 0.025 \\
\hline
\end{tabular}

Table 1: Tam model parameter values determined from experimental data or numerical calculations.

\section{III.C. Experimental Data}

The results of exploring the nondeterministic parameters in the shock-associated noise prediction models are compared to measurements consisting of noise data from supersonic jets issuing from a 2-inch exit diameter, circular, convergent nozzle. This nozzle was used in an extensive test program to measure the noise from jets at a variety of nozzle pressure and temperature ratios. ${ }^{4}$ The measured noise spectrum from this convergent 


\begin{tabular}{|c|c|c|c|c|c|c|c|c|c|}
\hline Case & $T_{t} / T_{\infty}$ & $P_{t} / P_{\infty}$ & $M_{j}$ & $U_{j} / c_{\infty}$ & $\beta$ & $\gamma_{j}$ & $T_{\infty}(\mathrm{K})$ & $P_{\infty}(\mathrm{Pa})$ & $c_{\infty}(\mathrm{m} / \mathrm{s})$ \\
\hline Cold & 1.01 & 3.636 & 1.493 & 1.245 & 1.109 & 1.4000 & 279.2 & 97790 & 334.93 \\
Hot & 2.16 & 3.629 & 1.494 & 1.828 & 1.109 & 1.3942 & 279.6 & 97839 & 335.18 \\
\hline
\end{tabular}

Table 2: Test conditions for a convergent nozzle used in the prediction models.

nozzle at a variety of operating conditions were recently compared to predictions from both jet mixing noise and jet shock-associated noise models. ${ }^{16}$ A subset of those conditions, one a cold jet and one a hot jet, are used in this study and are given in Table 2. Example spectral comparisons between measured and predicted shock-associated noise results at the operating conditions of Table 2 are shown in Figure 1.

\section{Results}

The results of this paper are divided into three parts. The first part discusses the effects of jet operating condition uncertainty on the predicted results. This is followed by a presentation of results where the uncertain parameters have known distributions. In the final part, the prediction model has many parameters with no clearly defined distributions. Estimates are made for these distributions in order to complete the analysis.

\section{IV.A. Operating Condition Input Parameter Uncertainty}

The validation of a prediction code is performed by comparing computed values with the same values obtained from an experimental measurement. For jet noise prediction, this comparison is done for various noise level metrics including overall and spectral levels at specific jet operating conditions. Hence, the operating conditions from the experiments are used as input to the jet noise prediction code. The primary experimental measurements are pressures, temperatures, and lengths or distances associated with the geometry of the nozzle and the location of measurement microphones. Any code specific operating condition input parameter, such as Mach number, can be computed using the measured values. The purpose here is to demonstrate the uncertainty of predicted noise levels given the known uncertainty in the experimental jet operating conditions. For this example, we will use the Tam model.

The ability to accurately measure pressures, temperatures, and lengths at the NASA Glenn Research Center aeroacoustic facilities has been documented in a recent assessment of noise prediction capability. ${ }^{17}$ Given those results, Table 3 shows an example of the measured operating conditions and the standard deviation $\sigma$ for the assumed normally distributed measured quantities. The table shows the measured total pressure and temperature, $P_{t}$ and $T_{t}$, the measured ambient pressure and temperature, $P_{\infty}$ and $T_{\infty}$, the measured nozzle exit diameter $D_{e}$, and the radial distance $R$ from the center of the nozzle exit area to the microphone. The example conditions are for the noise radiated from a jet issuing from a convergent nozzle with the hot jet operating conditions listed in Table 2 .

\begin{tabular}{|c|c|c|}
\hline Parameter & Measured Value (mean) & $\sigma$ \\
\hline$P_{t}$ & $355058 \mathrm{~Pa}$ & 139.3 \\
$T_{t}$ & $605.3 \mathrm{~K}$ & 1.89 \\
$P_{\infty}$ & $97839 \mathrm{~Pa}$ & 139.3 \\
$T_{\infty}$ & $279.6 \mathrm{~K}$ & 1.89 \\
$D_{e}$ & $0.0508 \mathrm{~m}$ & 0.000051 \\
$R$ & $5.08 \mathrm{~m}$ & 0.0127 \\
\hline
\end{tabular}

Table 3: Example measured jet operating conditions with standard deviations.

Following the Monte Carlo procedure given above, sampling calculations were performed from 100 to 10000 samples. In each case, the means and $95 \%$ confidence intervals were computed for the predicted 
shock-noise spectra at directivity angles from 50 to 90 degrees from the inlet direction. From these results, the maximum width and the average width confidence intervals were determined across all computed spectral frequencies and directivity angles and presented in Table 4. The increasing number of samples $N$ results in both more clearly defining the distribution of the computed solution and reducing the estimate of the standard error $\sigma / \sqrt{N}$, thereby reducing the confidence interval. The table clearly indicates that the effects of input parameter uncertainty on the predicted shock-associated noise, sound-pressure-level spectrum for the jet operating conditions listed in Table 3 are less than $0.2 \mathrm{~dB}$ at $95 \%$ confidence at the maximum and on average, less than $0.04 \mathrm{~dB}$. These levels of uncertainty would not be noticeable on spectral density plots such as those shown in Figure 1. In general, this shows that the ability to make accurate measurements of input parameters will lead to small uncertainties in the predicted values. This does not mean the predictions are accurate as can be seen in Figure 1 where many decibels of error can separate the predicted values from the measured values. Thus, we cannot rely on determining prediction uncertainty solely from the uncertainty in the jet operating condition input parameters, but must consider the uncertainty of the parameters within the model. We turn to these parameters, next.

\begin{tabular}{|c|c|c|}
\hline No. of Samples $N$ & Max. 95\% CI Width & Avg. 95\% CI Width \\
\hline 100 & $0.198 \mathrm{~dB}$ & $0.036 \mathrm{~dB}$ \\
200 & $0.146 \mathrm{~dB}$ & $0.025 \mathrm{~dB}$ \\
400 & $0.099 \mathrm{~dB}$ & $0.018 \mathrm{~dB}$ \\
1000 & $0.063 \mathrm{~dB}$ & $0.011 \mathrm{~dB}$ \\
10000 & $0.020 \mathrm{~dB}$ & $0.004 \mathrm{~dB}$ \\
\hline
\end{tabular}

Table 4: Maximum and average widths of spectral 95\% confidence intervals (CI) at upstream angles.

\section{IV.B. Uncertain Parameters with Known Distributions}

The sJet code computes the shock-associated noise spectrum by first computing an overall level value and then combining that value with a spectral function to produce the final results. For the convergent nozzle used here, the jet operating conditions and directivity angle set the spectral shape. The only uncertain values are $m$ and $B$ that were determined by regression and only enter the model prediction through the the overall level in the first two terms on the right hand side of equation (16). Hence, we compare the outputs using the uncertain parameters $m$ and $B$ in those two terms to the output obtained using the mean values $\bar{m}$ and $\bar{B}$. The spectrum result that would follow from equation (22) will have exactly the same level of uncertainty at all frequencies.

The linear regression analysis performed in Khavaran \& Bridges ${ }^{6}$ using equation (3) produced tables of $\bar{m}$ and $\bar{B}$ as a function of directivity angle and jet total temperature. In addition, the results of linear regression provide estimates for the standard deviations $\sigma_{m}$ and $\sigma_{B}$ and the correlation coefficient $\rho_{m B}$ between $m$ and $B$. These are also tabulated. The means and the standard deviations are used to define the normal distribution functions when the fixed parameters are replaced with nondeterministic parameters. If the correlation coefficient is nonzero, then the distribution function is jointly normal. For a deterministic calculation, the jet operating conditions are used as input to the sJet code and the code uses interpolation to select the appropriate $\bar{m}$ and $\bar{B}$ values from the tables. Since the jet operating conditions do not change in the nondeterministic calculation, additional random inputs must be provided to the code. To simplify the replacement of $\bar{m}$ and $\bar{B}$ with random values, normalized random variables are provided as input and a linear transformation is performed.

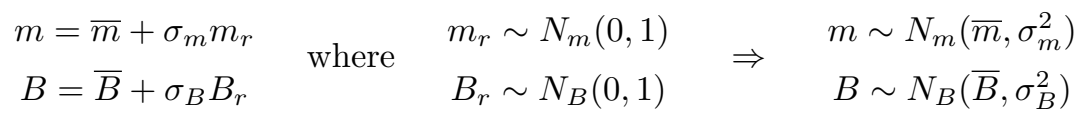

The symbol $N(0,1)$ represents a normally distributed random variable with a zero mean and a variance equal to one. The subscripts $m$ and $B$ on $N$ indicate that the random variables $m_{r}$ and $B_{r}$ are selected separately. The transformations in equation (8) provide the desired normal distributions for $m$ and $B$ where the jet operating conditions are used to select $\bar{m}, \bar{B}, \sigma_{m}$, and $\sigma_{B}$ by tabular interpolation. Up to this point, $m$ and 
$B$ have been considered to be uncorrelated. In the linear regression process that produced the tables for $\bar{m}$, and so forth, the correlation coefficient $\rho_{m B}$ was found to change very little and it was set at a constant value of 0.45 for all operating conditions and directivity angles. A constant $\rho_{m B}$ simplifies the introduction of a correlation between $m$ and $B$ into sJet. The normalized random variables $m_{r}$ and $B_{r}$ are sampled external to $s J e t$ with a fixed correlation and when provided to sJet the transformation maintains that correlation in the resulting $m$ and $B$ parameters.

Following the Monte Carlo procedure, the random inputs are provided to the sJet code by DAKOTA using the latin hypercube sampling technique. The outputs from the sJet code are then stored and analyzed by DAKOTA. The first set of results compare the amplitude level given by equation (3) with fixed parameters determined by the linear regression to the amplitudes computed with nondeterministic parameters. Figure 2 is a summary of results for the hot jet case at a directivity angle of 50 degrees where $\bar{m}=5.75$ and $\bar{B}=117.76$. The figure shows the mean levels and the $95 \%$ confidence intervals as a function of the number of random samples. The single point on the right is an example result after 10000 samples. In addition, the effects of changing $\sigma_{m}$ and $\sigma_{B}$ from half to twice their original tabulated value without and with correlation are shown. The following results are deduced from examining Figure 2:

1. The mean value of the output distribution, here $120.5 \mathrm{~dB}$, is basically unaffected by the width of the distributions of $m$ and $B$, by whether or not there is any correlation between $m$ and $B$, and by the number of samples. This is the expected result when the output level (in $\mathrm{dB}$ ) is simply treated as a number in a linear calculation.

2. Wider distributions in the input parameters leads to a wider distribution in the output. Again, this is due to the linear calculation.

3. The width of the confidence intervals decreases as the number of samples $N$ increases, as expected, since it varies as $1 / \sqrt{N}$. For the given $\sigma_{m}$ and $\sigma_{B}$ and no correlation, the confidence interval is $\pm 0.39 \mathrm{~dB}$ wide at 100 samples decreasing to $\pm 0.12 \mathrm{~dB}$ wide at 1000 samples. Since this is a result of a linear calculation, the confidence interval is symmetric about the mean value.

4. The presence of correlation between $m$ and $B$ increases the width of the output confidence interval. On a dB scale, this was not significant as the increase was less than $0.1 \mathrm{~dB}$.

The alternative to treating the decibel output as a number in an uncertainty calculation is to recognize the nonlinear nature of the power level calculation and perform the uncertainty calculation using equation (6). Using the same Monte Carlo approach with the normalized random input variables as above, the mean power levels and the $95 \%$ confidence intervals are computed before converting the results to decibels. Figure 3 is the equivalent plot to Figure 2 for the nonlinear approach. The mean parameters for the hot jet case at a directivity angle of 50 degrees are $\bar{m}=5.60$ and $\bar{E}=11.505$. Using these as fixed parameters results in an output power level of $117.7 \mathrm{~dB}$. The results for the nondeterministic calculations show the following:

1. The mean of the output level depends on the output distribution generated by the distributions of the random parameters $m$ and $E$. Both the size of the parameter distributions and whether the parameters are correlated affect the output. The fact that the mean output level increases with increasing $\sigma$ follows directly from the fact that any random change in the parameters that increases the power level of the output will increase the mean power in a quadratic summation of all sampled power levels.

2. Using latin hypercube sampling, more samples results in a better definition of the output distribution and convergence in the statistical values computed from that distribution. Hence the mean output levels reach an asymptote with an increasing number of samples.

3. The $95 \%$ confidence intervals are in general not symmetric about the mean value. Given $\sigma_{m}, \sigma_{E}$, and no correlation, the confidence interval is $+0.34 \mathrm{~dB},-0.37 \mathrm{~dB}$ at 100 samples decreasing to a confidence interval of $+0.11 \mathrm{~dB},-0.12 \mathrm{~dB}$ at 1000 samples.

4. Including the effects of a correlation between parameters $m$ and $E$ results in an increase in the mean output levels and width of the $95 \%$ confidence intervals. In terms of decibels, these increases are small for the average correlation coefficient $\rho_{m E}=0.43$, up to $0.2 \mathrm{~dB}$ in the mean level and up to $0.08 \mathrm{~dB}$ in the confidence interval width at 1000 samples. 


\section{IV.C. Many Uncertain Parameters with Unknown Distributions}

The previous model had two uncertain parameters with clearly defined distributions determined from regressions that fit model equations to measured data. We now consider the Tam model, equation (7), that contains 12 parameters, listed in Table 1, that each have some level of uncertainty. To determine the effects of these uncertainties, we have to define the probability distributions in order to replace the fixed parameters with distributions. For the Tam model, the detailed information required to make such distributional specifications is not given in the references describing the development of that model. A parameter for which there is information available to estimate a probability distribution is the $v_{c 1}$ parameter that is used to determine the convective velocity ratio $U_{c} / U_{j}$. The data given in Harper-Bourne \& Fisher ${ }^{18}$ and Harper-Bourne, ${ }^{19}$ have been used to determine a fixed value for $v_{c 1}$ of about 0.7 . That same data is used here to determine a mean value of 0.708 and a variance of 0.00937 for both a normal distribution and an equivalent uniform distribution. The uniform distribution covers a range from 0.54 to 0.876 or about $\pm 24 \%$ from the mean value. Since the data were insufficient to know the exact behavior of the $v_{c 1}$ distribution, both the normal and the uniform distributions were applied to replace the fixed parameter. The DAKOTA code, using the latin hypercube sampling technique, performed 100 samples from the $v_{c 1}$ parameter distribution and computed the mean and the $95 \%$ confidence interval (CI) using the pressure-squared output distribution from the Tam model. The remaining parameters were fixed at the values given in Table 1 . The results are shown in Figure 4 using the cold jet conditions at a 50 degree inlet directivity angle. For all but the lowest frequency results, the spectra generated using the nondeterministic $v_{c 1}$ parameter with either the normal distribution or the uniform distribution are essentially the same. Only 24 frequencies were used in the nondeterministic calculations, hence the mean and $95 \%$ CI spectra are not as smooth as the fixed parameter spectrum which was evaluated in narrow band with a $40 \mathrm{~Hz}$ bandwidth.

There are 11 other parameters in the Tam model that were identified as being replaceable by distributions. Without any specific information about these parameters, we estimate a distribution for each parameter based on the $v_{c 1}$ distribution results in Figure 4. First, the difference between using a normal or a uniform distribution was small. Second, the uniform distribution had a range based on the mean value of $v_{c 1} \pm 24 \%$. Observation of the $v_{c 1}$ data indicates that this range was perhaps too wide. We reduce the range by about half or $\pm 10 \%$. Considering this to be conservative, we apply this to all the parameters listed in Table 1 and use their listed values to define uniform distributions with a range given by their individual mean value $\pm 10 \%$. A result of a 100-sample calculation is shown in Figure 5a for the hot jet case at a directivity angle of 50 degrees where all 12 parameters are nondeterministic and varied independently. This result shows that the predicted spectrum using the mean parameter values (that is the original model fixed values, shown by the red line) is not the same as the mean spectrum of an ensemble of spectra computed using model parameters whose mean values of the distributions are the same as the original fixed parameters. The variances or distributions of the uncertain model parameters have affected the output distribution that was used to compute the mean spectrum. To emphasize this, Figure 5b shows an expanded view of the main spectral peak where both the number of random samples and the range of the uniform distribution where changed. The increased number of samples provides a better definition of the output distribution. This does not change the output mean value significantly but does reduce the size of the confidence interval by $1 / \sqrt{N}$ as mentioned before. Reducing the variance of the nondeterministic parameter distributions by reducing the range from $\pm 10 \%$ to $\pm 2 \%$ does show the points of the mean spectrum approaching the fixed parameter spectrum.

The results in Figure 5a for the output variance from the Tam model, as expressed in the displayed confidence interval, when 12 parameters were varied using uniform distributions are similar to the 1 parameter distribution results shown in Figure 4. The confidence intervals shown in both figures are about $0.5 \mathrm{~dB}$ wide at the peak. The $v_{c 1}$ parameter with the wider distribution has given as much output variance as all 12 parameters give with smaller distributions. By itself, the $v_{c 1}$ parameter with a uniform $\pm 10 \%$ distribution results in a peak frequency confidence interval width of $0.12 \mathrm{~dB}$. The output confidence interval for each of the other 11 parameters, computed separately, range from 0 to $0.68 \mathrm{~dB}$ in width at the peak. This indicates that the uncertainty in each parameter does not have the same influence on the output uncertainty and that computing output uncertainty due to individual parameter uncertainty neglects any interaction between parameters. To determine which parameters do not have significant influence and to include the effects of interactions between parameters, the variance-based decomposition technique is used to compute the total effect sensitivity measure given by equation (1). All 12 parameters have uniform distributions with a range of $\pm 10 \%$ from the mean value. A result of this analysis is shown in Figure 6a for the cold jet case at a 
directivity angle of 50 degrees. The figure shows the $S_{T i}$ values for each parameter added on top of each other as a function of the jet Strouhal number. The width of the $S_{T i}$ band is related to the influence of the parameter on the output variance. Thus, non-influential parameters have small total effect sensitivity measures.

Figure 6b shows the nondeterministic parameter spectra compared to the fixed parameter spectrum and the measured data that corresponds to the results in Figure 6a. Comparing these two figures, we see in the region of the major peak that 9 of the 12 parameters have small total effect measures. The important parameters are those associated with the convected velocity $\left(v_{c 1}\right)$, the lowest mode wave number of the shock cell structure model $\left(w_{11}\right)$, and the half-width of the similarity source $\left(h_{s 1}\right)$. From the results in the region of the second peak, we include the parameter associated with the second mode wave number of the shock cell structure model $\left(w_{21}\right)$ as being an additional influential parameter. Fixing the other 8 parameters, the 100-sample calculation with the Tam model was repeated. The resulting spectra are also shown in Figure $6 \mathrm{~b}$. The sizes of the output spectral variance are about the same between the 12-parameter and the 4-parameter distribution models. Thus, the sensitivity analysis has identified 8 relatively non-influential parameters in the prediction model.

The same set of plots is shown in Figure 7 for the 90 degree inlet directivity angle. The peak frequency of the spectra have moved to a higher Strouhal number of 0.6, and the total effect sensitivity measures reflect this shift. The same 4 parameters as found at 50 degrees have the most influence and only the 4 parameter nondeterministic spectra are shown compared to the fixed parameter spectrum and the measured data. The 50 degree and 90 degree sensitivity results are shown in Figures 8 and 9, respectively, for the hot jet case. These results do not show any significant changes in the influential parameters especially in the peak regions of the spectrum. In all these results, only the $h_{s 1}$ parameter has influence on amplitude uncertainty in the peak frequency region. The other amplitude parameters such as $c_{b 1}$ and $a_{b 1}$ only have their most influence on the output uncertainty at the higher frequencies. The parameters with consistent little influence are $x_{c 1}$ and $x_{c 2}$ that are related to the jet potential core length model and $v_{c 2}$ which attempts to include temperature effects in the model for convective velocity.

Finally, we consider whether using nondeterministic parameters leads to better predictions. An examination of the spectral results in Figures 6 to 9 shows that the mean spectrum computed after sampling 4 nondeterministic parameters with uniform distributions have lowered peak levels and increased valley levels. At 50 degrees, the primary and secondary peaks have broadened due to the interaction of the changing wave numbers and the source width. This broadening reduces peak-to-valley levels. In addition, at 90 degrees the peak frequency of the mean spectrum shifts to a frequency lower than the fixed spectrum peak frequency and has better agreement with the measured peak frequency. These changes appear relatively small for the added complication of a nondeterministic calculation compared to the fixed parameter calculation. However, the total uncertainty of the Tam model output cannot be determined without varying its internal parameters. Consider the 4 parameters with the most influence on output spectrum uncertainty. Only the source width related parameter $h_{s 1}$ was determined from a fit to acoustic data. Its influence is both through the mode summation in equation (7) that determines spectral shape and directly as an amplitude parameter. The other parameters $v_{c 1}, w_{11}$, and $w_{21}$ were determined in stability and waveguide models related to the jet flow. The true level of uncertainty in these 4 parameters is unknown and small changes in these parameters affect the output spectrum. If we sample these parameters using the $\pm 10 \%$ uniform distribution and plot the possible spectral outputs, then after 100 samples results such as those shown in Figure 10 are obtained. A widely scattered set of spectra are shown where the fixed parameter spectrum is but one of the realized spectra. The best alternative to choosing which parameter set is best is to account for the distribution of the model parameters, compute the distribution of the output, and take the mean of the output distribution with its known level of uncertainty as your prediction. This result has not necessarily given a better prediction method, compared to any particular set of measured data, but it does provide a quantified level of uncertainty for the prediction and the most influential parameters were identified that may aid in developing an improved model.

\section{Concluding Remarks}

Engineering applications in aircraft noise prediction rely on modeling of complex physical phenomena in order to obtain solutions rapidly for design, development, and parametric studies. Parameters within these models are usually fixed in value even though there are uncertainties related to those values. It may be more 
appropriate to allow a parameter to cover a range of values by replacing a fixed value with a distribution of values. A nondeterministic calculation must then be performed. In this paper, a Monte Carlo method was used. A random sample from one or more parameter distributions was chosen, processed by the prediction model, and the output value recorded. This process was repeated until a distribution of output values was obtained. From this output distribution, mean and confidence interval values were computed representing the predicted solution and its uncertainty.

The above nondeterministic calculation process was applied to two models for the prediction of supersonic jet broadband shock-associated noise. The first model, called the sJet code, was developed from a study of far-field spectral data. It contained two nondeterministic parameters with statistical properties derived from performing both linear and nonlinear regression. These parameters only affect the overall level of the prediction and not its spectral shape. Using regression, the statistical properties of the parameters were obtained and used to define the parameter's normal distribution. Random samples from these distributions were used to compute the output acoustic power levels. When averaging a large number of acoustic power levels, random higher values will raise the average level. Only the results using nondeterministic parameters derived from the nonlinear regression approach exhibited this behavior.

The second model, the Tam model, was developed from a derivation of the equations of motion followed by using physical models to simplify the calculations. This model was used to demonstrate that the small uncertainty in high quality measurements of the jet operating conditions used as code input yielded small uncertainties in the code output. This is insufficient for demonstrating the model's output uncertainty and the uncertainty of internal model parameters must be taken into account. This model contained 12 internal nondeterministic parameters for which estimates had to be made of their distributions in order to complete the analysis. External reference data were used to estimate the $v_{c 1}$ parameter distribution. It was found that the output spectrum confidence interval was not changed much between using a normal distribution and a uniform distribution. Based on that result, all parameters were assigned a uniform distribution but with a smaller variance. A global sensitivity analysis performed with these 12 nondeterministic parameters identified 8 non-influential parameters. Using the remaining 4 influential nondeterministic parameters, calculations were performed to judge the benefit of this approach for predictive capability. The results showed that the fixed parameter spectral result is only one possible solution and that the mean spectral result with its accompanying confidence interval computed from a distribution of output spectra would be the preferred result. This is the best way to quantify the uncertainty in the prediction from this model.

\section{Appendix A - sJet Model Spectrum Calculation}

The sJet code computes the far field radiated noise spectra from subsonic and supersonic jets. Both the mixing noise and the broadband shock-associated noise spectra, as appropriate, are computed as a function of the inlet directivity angle. For supersonic jets, the predicted radiated noise is modified depending on whether the jet issued from a convergent or a convergent-divergent nozzle. In addition, if screech is present, there is the ability to include noise amplification effects in the predicted spectra. The details of the modeling are found in Khavaran \& Bridges. ${ }^{4,6}$ Here, only the equations for computing the radiated broadband shockassociated noise spectra are given. The formulation is a modification of the model given by Deneuville ${ }^{20}$ and Gliebe et al. ${ }^{21}$

The following parameters are defined:

$\beta$ - Shock parameter

$$
\beta=\sqrt{\left|M_{j}^{2}-M_{d}^{2}\right|}
$$

where $M_{j}$ is the fully expanded jet Mach number and $M_{d}$ is the nozzle design Mach number with $M_{d}=1$ for a convergent nozzle.

$\beta_{m}$ - Modified shock parameter for convergent-divergent nozzles, $\beta_{m}=\beta$ for convergent nozzles. For $M_{d}>1.01, \beta_{m}$ depends on whether the nozzle pressure ratio $P_{t} / P_{\infty}$ is greater or less than the critical pressure ratio.

$$
\beta_{m}= \begin{cases}\left(\beta M_{d}^{3 / 2}\right)^{M_{d}^{-3 / 2}}, \frac{P_{t}}{P_{\infty}}>\frac{P_{c r i t}}{P_{\infty}}, \text { under-expanded } \\ \beta^{M_{d}^{-3 / 2}}, \frac{P_{t}}{P_{\infty}}<\frac{P_{c r i t}}{P_{\infty}} \text {, over-expanded }\end{cases}
$$


$L_{a v g}$ - The average spacing length between shock cells in the jet shock cell structure.

$$
L_{a v g}=1.1 D_{e} \sqrt{M_{j}^{2}-1}
$$

where $D_{e}$ is the nozzle exit diameter.

$U_{c}, M_{c}-$ Convective velocity and Mach number

$$
\begin{aligned}
U_{c} & =0.7\left(U_{j}-U_{\infty}\right) \\
M_{c} & =\frac{U_{c}}{c_{\infty}}
\end{aligned}
$$

$C_{u o}-$ Sound level correction for convergent-divergent nozzles, $M_{d}>1$

$$
C_{u o}= \begin{cases}-10\left\{1.04+\tanh \left[4\left(\beta_{m}-1.49\right)\right]\right\} & , M_{j}>M_{d} \\ -15\left\{1.0+\tanh \left[7.9\left(\frac{\beta^{2}}{M_{d}^{2}}-0.379\right)\right]\right\}, & M_{j}<M_{d}\end{cases}
$$

$\theta_{c}-$ Critical angle

$$
\theta_{c}= \begin{cases}\pi & , M_{c} \leq 1 \\ \pi-\tan ^{-1}\left(\sqrt{M_{c}^{2}-1}\right), & M_{c}>1\end{cases}
$$

For each inlet directivity angle $\theta_{\ell}, \ell=1,2, \ldots, N_{a n g}$, compute the shock-associated noise spectrum if $\theta_{\ell}<\theta_{c}$. First set the maximum sound pressure level

$$
\operatorname{SPLMAX}_{\ell}=10 m_{\ell} \log \beta_{m}+B_{\ell}+42.37+10 \log \left\{\frac{N_{s}}{8} \frac{\pi}{4}\left(\frac{D_{e}}{R}\right)^{2} \frac{1}{\left(1-\frac{U_{\infty}}{c_{\infty}} \cos \theta_{\ell}\right)^{4}}\right\}
$$

where $N_{s}$ is the number of shock cells (typically $N_{s}=8$ ), $R$ is the radial distance from the nozzle to the microphone, and $m_{\ell}$ and $B_{\ell}$ are determined by the linear regression given by equation (3). If the nozzle is convergent-divergent, then modify $\mathrm{SPLMAX}_{\ell}$ by

$$
\operatorname{SPLMAX}_{\ell}=\mathrm{SPLMAX}_{\ell}+C_{u o} \quad M_{j}>M_{d} \text {. }
$$

The fundamental frequency of the peak of the shock-associated noise spectrum is given by

$$
f_{p, \ell}=\frac{U_{c}}{L_{a v g}\left(1+M_{c} \cos \theta_{\ell}\right)\left(1-\frac{U_{\infty}}{c_{\infty}} \cos \theta_{\ell}\right)} .
$$

Finally, compute the spectrum for each frequency for $k=1,2, \ldots, K$.

$$
\begin{gathered}
\xi_{k \ell}=\frac{f_{k}}{f_{p, \ell}} \\
S_{1}=\operatorname{SPLMAX}_{\ell}+10 \log \frac{U_{j}}{D_{e} f_{p, \ell}}+\left\{\begin{array}{l}
10 \log \left(\xi_{k \ell}\right)^{7}, \xi_{k \ell} \leq 1 \\
10 \log \left(\xi_{k \ell}\right)^{-4}, \xi_{k \ell}>1
\end{array}\right. \\
S_{2}=\operatorname{SPLMAX}_{\ell}-6+10 \log \frac{U_{j}}{D_{e} f_{p, \ell}}+\left\{\begin{array}{l}
10 \log \left(\xi_{k \ell} / 2\right)^{2}, \xi_{k \ell} \leq 2 \\
10 \log \left(\xi_{k \ell} / 2\right)^{-2}, \xi_{k \ell}>2
\end{array}\right. \\
\operatorname{SPL}_{k \ell}=10 \log \left\{10^{S_{1} / 10}+10^{S_{2} / 10}\right\}
\end{gathered}
$$




\section{Appendix B - Weighting in Regression Analysis}

The necessity to use weighting in the nonlinear regression formulation given in equation (6) is due to the wide range of values found for $y_{i}=\left\langle p^{2}\right\rangle_{s h}$. The acoustic power levels considered here cover many orders of magnitude.

The residual error is related to the variation in the data value $y_{i}$ minus the estimated value $\widehat{y}_{i}$ from the nonlinear regression. At a particular data point after the fit, $y_{i}$ is a constant and the variation in the residual error $\epsilon_{i}=y_{i}-\widehat{y}_{i}$ will follow the variation in $\widehat{y}_{i}$. Using the analysis in Bendat \& Piersol, ${ }^{14}$ we construct an error band for the data value $y_{i}$ based on the confidence interval for an averaged spectrum. Let the following probability interval for the spectral density $G_{p p}$

$$
\operatorname{Pr}\left[\frac{n}{\chi_{n ; \alpha / 2}^{2}} \widehat{G}_{p p}<G_{p p}<\frac{n}{\chi_{n ; 1-\alpha / 2}^{2}} \widehat{G}_{p p}\right]=1-\alpha
$$

be the definition of a $(1-\alpha) 100 \%$ confidence interval for an averaged spectral density $\widehat{G}_{p p}(f)$ where $\widehat{G}_{p p}$ has a chi-squared distribution with $n$-degrees of freedom and $\chi_{n ; \alpha}^{2}$ is the $100 \alpha$ percentage point of the $\chi^{2}$ distribution. The total acoustic power is then computed as the sum of all components in the spectrum

$$
\left\langle p^{2}\right\rangle=\sum_{k=1}^{K} \widehat{G}_{p p}\left(f_{k}\right)
$$

which is distributed as $\chi_{K \times n}^{2}$. The confidence interval for $\left\langle p^{2}\right\rangle$ is also determined by equation (23) with $(K \times n)$-degrees of freedom. The size of the variation in the data value is given by

$$
\Delta\left\langle p^{2}\right\rangle=\frac{K \times n}{\chi_{K \times n ; 1-\alpha / 2}^{2}}\left\langle p^{2}\right\rangle-\frac{K \times n}{\chi_{K \times n ; \alpha / 2}^{2}}\left\langle p^{2}\right\rangle .
$$

Thus, if the variance of the residual error

$$
\operatorname{Var}\left[\epsilon_{i}\right] \propto \Delta\left\langle p^{2}\right\rangle_{i}
$$

then by using weighting and applying equation (25)

$$
\frac{\epsilon_{i}}{\sqrt{y_{i}}}=\frac{y_{i}-\widehat{y}_{i}}{\sqrt{y_{i}}} \Rightarrow \operatorname{Var}\left[\frac{\epsilon_{i}}{\sqrt{y_{i}}}\right]=\frac{\operatorname{Var}\left[\epsilon_{i}\right]}{y_{i}} \propto \frac{\Delta\left\langle p^{2}\right\rangle_{i}}{\left\langle p^{2}\right\rangle_{i}}=\text { constant } .
$$

By using the weighting given by equation (27), the variances of the residual errors are now equal as desired for performing the nonlinear regression using equation (6). Furthermore, the distribution of the residual error is nominally $\chi^{2}$ but it approaches a normal distribution for a large number of degrees of freedom. For the overall power level computed from a typical power spectral density with 6000 frequency bins averaged 400 times, the number of degrees of freedom is $2 \times 400 \times 6000=4.8 \times 10^{6}$.

\section{Appendix C - Tam Model Detailed Equations}

The parametric form of the detailed model equations for the Tam broadband shock-associated noise prediction model, given in equation (7), are presented in this appendix. These equations follow from those found in Tam. ${ }^{5}$

$\bar{c}$ - An amplitude factor where $c_{b 1}$ was determined by fitting the far field spectrum to measured data with $S t_{r e f}=0.35$.

$$
\bar{c}\left(c_{b 1}\right)=c_{b 1} \times S t_{r e f}
$$

$L$ - The source model includes a representation of growing and decaying instability waves. $L$ is the half-width of a Gaussian function that replaces the amplitude envelope of the instability wave. The coefficient $h_{s 1}$ was determined by fitting the far field spectrum to measured data. $M_{j, r e f}=1.67$ is a reference Mach number.

$$
L\left(h_{s 1}, x_{c 1}, x_{c 2}\right)=h_{s 1} \frac{x_{c}\left(x_{c 1}, x_{c 2}, M_{j}\right)}{x_{c}\left(x_{c 1}, x_{c 2}, M_{j, r e f}\right)}
$$


$x_{c}-\mathrm{A}$ model for the jet potential core length. The coefficients $x_{c 1}$ and $x_{c 2}$ were determined by fitting a line to measured mean flow data.

$$
x_{c}\left(x_{c 1}, x_{c 2}, M_{j}\right)= \begin{cases}{\left[x_{c 1}+x_{c 2}\left(M_{j}^{2}+1-\frac{T_{j}}{T_{\infty}}\right)\right] D_{j},} & \frac{T_{j}}{T_{\infty}} \leq 1 \\ \left(x_{c 1}+x_{c 2} M_{j}^{2}\right) D_{j} & , \frac{T_{j}}{T_{\infty}}>1\end{cases}
$$

$D_{j}$ - The diameter of the fully expanded jet derived by using mass conservation.

$$
D_{j}=D_{e}\left[\left(1+\frac{\gamma-1}{2} M_{j}^{2}\right)\left(1+\frac{\gamma-1}{2} M_{d}^{2}\right)^{-1}\right]^{\frac{\gamma+1}{4(\gamma-1)}}\left(\frac{M_{d}}{M_{j}}\right)^{1 / 2}
$$

$\bar{A}^{2}$ - An amplitude quantity that relates to the shock cell strength. The coefficients $a_{b 1}$ and $a_{b 2}$ were determined to provide a best fit to acoustic data.

$$
\bar{A}^{2}\left(a_{b 1}, a_{b 2}\right)=\left\{\begin{array}{l}
\frac{A_{1}^{2}\left(\frac{D_{e}}{D_{j}}\right)^{2}}{1+a_{b 1} A_{1}^{a_{b 2}}}, \frac{M_{j}}{M_{d}} \geq 1 \\
\frac{A_{1}^{2}}{1+a_{b 1} A_{1}^{a_{b 2}}}, \frac{M_{j}}{M_{d}}<1
\end{array}\right.
$$

where

$$
A_{1}=\frac{\left|M_{j}^{2}-M_{d}^{2}\right|}{1+\frac{\gamma-1}{2} M_{d}^{2}}
$$

$f_{\nu}$ - The peak frequency of the spectrum associated with the $\nu$-th waveguide mode of the shock cell structure.

$$
f_{\nu}\left(v_{c 1}, v_{c 2}, w_{11}, w_{12}, w_{21}, w_{\nu 1}\right)=\frac{U_{c}\left(v_{c 1}, v_{c 2}\right) k_{\nu}\left(w_{11}, w_{12}, w_{21}, w_{\nu 1}\right)}{2 \pi}\left(1+M_{c}\left(v_{c 1}, v_{c 2}\right) \cos \theta\right)^{-1}
$$

$k_{\nu}$ - The wave number for the $\nu$-th waveguide mode of the shock cell structure. The coefficients $w_{11}, w_{12}$, $w_{21}$, and $w_{\nu 1}$ were chosen to provide better agreement with multiple-scales shock cell calculations.

$$
k_{\nu}\left(w_{11}, w_{12}, w_{21}, w_{\nu 1}\right)= \begin{cases}1.1\left(w_{11}-w_{12} M_{j}\right) k_{1, v s}, & \nu=1 \\ w_{21} k_{2, v s} & , \nu=2 \\ w_{\nu 1} k_{\nu, v s} & , \nu>2\end{cases}
$$

where $k_{\nu, v s}$ is the wave number for the vortex sheet shock cell structure and $j_{0, \nu}$ is the $\nu$-th zero of the zeroth order Bessel function.

$$
k_{\nu, v s}=\frac{2 j_{0, \nu}}{D_{j} \sqrt{M_{j}^{2}-1}}
$$

$U_{c}$ - The convective velocity of large turbulent structures in the jet. The coefficients $v_{c 1}$ and $v_{c 2}$ were determined from stability calculations

$$
U_{c}\left(v_{c 1}, v_{c 2}\right)= \begin{cases}v_{c 1} U_{j} & , \frac{T_{t}}{T_{\infty}} \leq 1 \\ {\left[v_{c 1}-v_{c 2}\left(\frac{T_{t}}{T_{\infty}}-1\right)\right] U_{j},} & \frac{T_{t}}{T_{\infty}}>1\end{cases}
$$

and the convective Mach number follows.

$$
M_{c}\left(v_{c 1}, v_{c 2}\right)=\frac{U_{c}\left(v_{c 1}, v_{c 2}\right)}{c_{\infty}}
$$




\section{References}

${ }^{1}$ Swiler, L. P., Adams, B. M., and Eldred, M. S., "Model Calibration under Uncertainty: Matching Distribution Information," AIAA Paper No. 2008-5944, 2008.

${ }^{2}$ Noor, A. K., "Perspectives on Nondeterministic Approaches," Nondeterministic Approaches and Their Potential for Future Aerospace Systems, edited by A. K. Noor, NASA CP-2001-211050, 2001, pp. 2 - 36.

${ }^{3}$ Bridges, J. E., Khavaran, A., and Hunter, C., "Assessment of Current Jet Noise Prediction Capabilities," AIAA Paper No. 2008-2933, 2008.

${ }^{4}$ Khavaran, A. and Bridges, J., "SHJAR Jet Noise Data and Power Spectral Laws," NASA TM-2009-215608, 2009.

${ }^{5}$ Tam, C. K. W., "Broadband Shock-Associated Noise of Moderately Imperfectly Expanded Supersonic Jets," J. Sound Vib., Vol. 140, No. 1, 1990, pp. 55-71.

${ }^{6}$ Khavaran, A. and Bridges, J., "Development of Jet Noise Power Spectral Laws Using SHJAR Data," AIAA Paper No. 2009-3378, 2009.

${ }^{7}$ Morris, P. J. and Miller, S. A. E., "The Prediction of Broadband Shock-Associated Noise Using RANS CFD," AIAA Paper No. 2009-3315, 2009.

${ }^{8}$ Eldred, M. S., Adams, B. M., Gay, D. M., Swiler, L. P., Haskell, K., Bohnhoff, W. J., Eddy, J. P., Hart, W. E., Watson, J.-P., Hough, P. D., and Kolda, T. G., "DAKOTA, A Multilevel Parallel Object-Oriented Framework for Design Optimization, Parameter Estimation, Uncertainty Quantification, and Sensitivity Analysis: Version 4.2 User's Manual," Sandia Technical Report SAND2006-6337, Sandia National Laboratory, October 2006, Updated November 6, 2008.

${ }^{9}$ Thacker, B. H., Riha, D. S., Millwater, H. R., and Enright, M. P., "Errors and Uncertainties in Probabilistic Engineering Analysis," AIAA Paper No. 2001-1239, 2001.

${ }^{10}$ Saltelli, A., Ratto, M., Tarantola, S., and Campolongo, F., "Sensitivity Analysis Practices: Strategies for Model-Based Inference," Reliability Engineering and System Safety, Vol. 91, 2006, pp. 1109-1125.

${ }^{11}$ Saltelli, A., Ratto, M., Andres, T., Campolongo, F., Cariboni, J., Gatelli, D., Saisana, M., and Tarantola, S., Global Sensitivity Analysis. The Primer, John Wiley, Chichester, Eng., 2008.

${ }^{12}$ Ratkowski, D. A., Nonlinear Regression Modeling: A Unified Practical Approach, Marcel Dekker, New York, NY, 1983.

${ }^{13}$ Bates, D. M. and Watts, D. G., Nonlinear Regression Analysis and Its Applications, Wiley, New York, NY, 1988.

${ }^{14}$ Bendat, J. S. and Piersol, A. G., Random Data Analysis and Measurement Procedures, Wiley-Interscience, New York, NY, 2nd ed., 1986.

${ }^{15}$ Tam, C. K. W., "Forward Flight Effects on Broadband Shock Associated Noise of Supersonic Jets," AIAA Paper No. 89-1088, 1989.

${ }^{16}$ Dahl, M. D., "Predictions of Supersonic Jet Mixing and Shock-Associated Noise Compared with Measured Far-Field Data," NASA TM-2010-216328, 2010.

${ }^{17}$ NASA, Assessment of NASA's Aircraft Noise Prediction Capability, TP-20XX-215653, to be published.

${ }^{18}$ Harper-Bourne, M. and Fisher, M. J., "The Noise from Shock Waves in Supersonic Jets," Noise Mechanisms, AGARDCP-131, 1974, pp. 11.1-11.13.

${ }^{19}$ Harper-Bourne, M., "On Modelling the Near-Field Noise of the High-Speed Jet Exhausts of Combat Aircraft," AIAA Paper No. 2002-2424, 2002.

${ }^{20}$ Deneuville, P., "Simplified Prediction Method of Shock Cell Noise of Supersonic Jets from Conical Nozzles," SNECMA YKA No. 5982/76, Societe Nationale d'Edute et de Construction de Moteurs d'Avion, France, 1976.

${ }^{21}$ Gliebe, P. R., Motsinger, R. E., and Sieckman, A., "High Velocity Jet Noise Source Location and Reduction. Task 6. Supplement. Computer Programs: Engineering Correlation (MS) Jet Noise Prediction Method and Unified Aeroacoustic Prediction Model (M*G*B) for Nozzles of Arbitrary Shape; Final Report," FAA-RD-76-79-6A, FAA, 1979. 


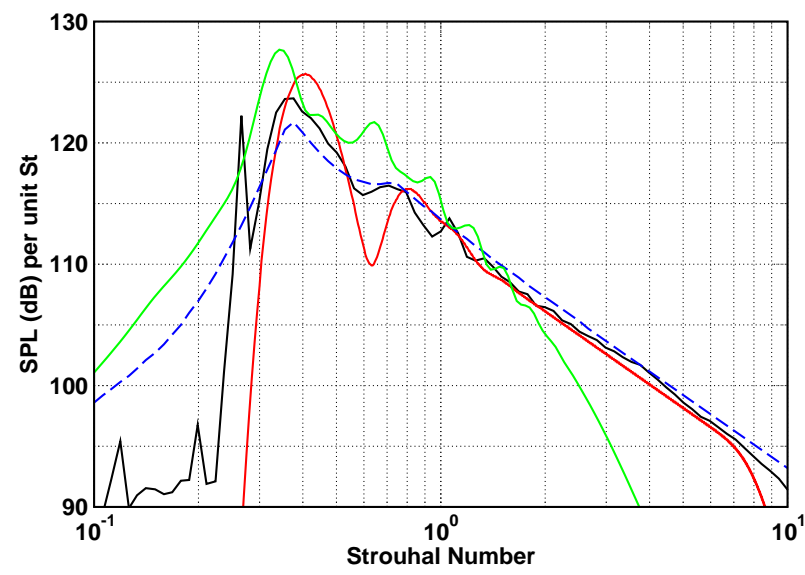

(a) Power spectral density at 50 deg., $M_{j}=1.493$, $P_{t} / P_{\infty}=3.636, T_{t} / T_{\infty}=1.01$

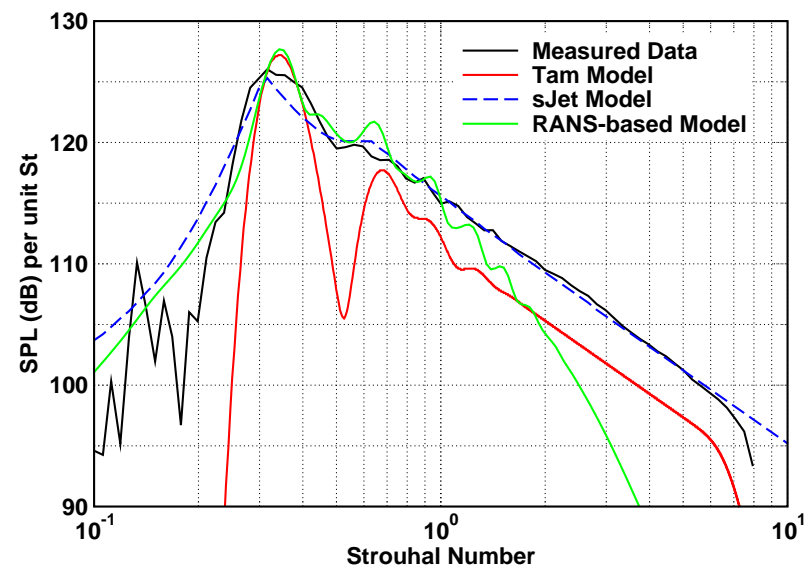

(c) Power spectral density at $50 \mathrm{deg}$., $M_{j}=1.494$, $P_{t} / P_{\infty}=3.629, T_{t} / T_{\infty}=2.16$

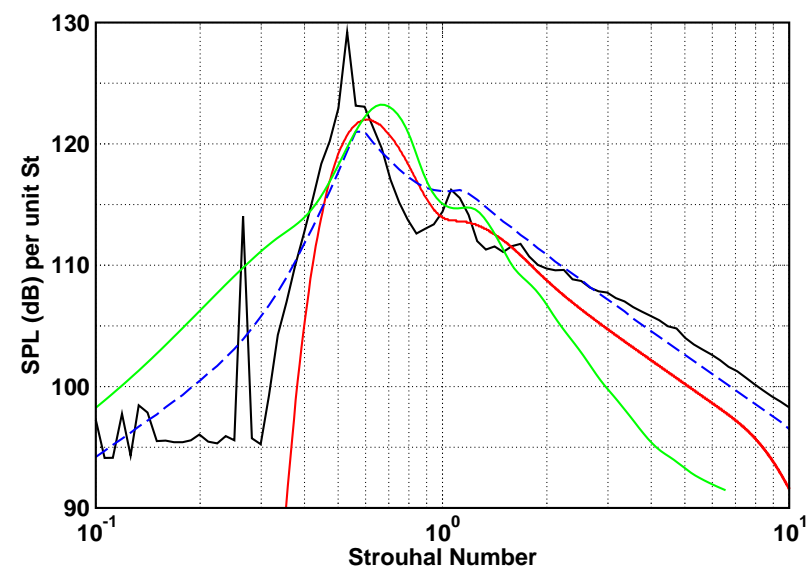

(b) Power spectral density at 90 deg., $M_{j}=1.493$, $P_{t} / P_{\infty}=3.636, T_{t} / T_{\infty}=1.01$

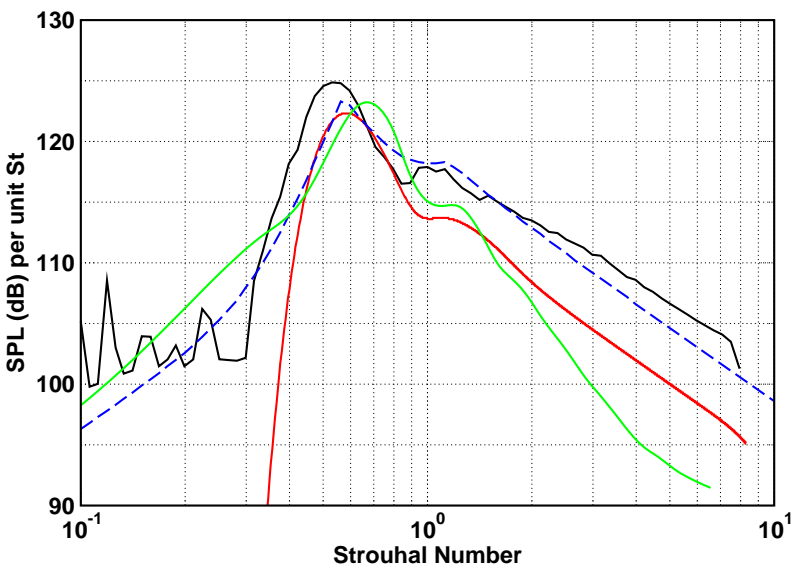

(d) Power spectral density at 90 deg., $M_{j}=1.494$, $P_{t} / P_{\infty}=3.629, T_{t} / T_{\infty}=2.16$

Figure 1: Power spectral densities from 3 shock-associated noise prediction models compared to the shockassociated noise component of measured data, distance $100 D_{e}$. 


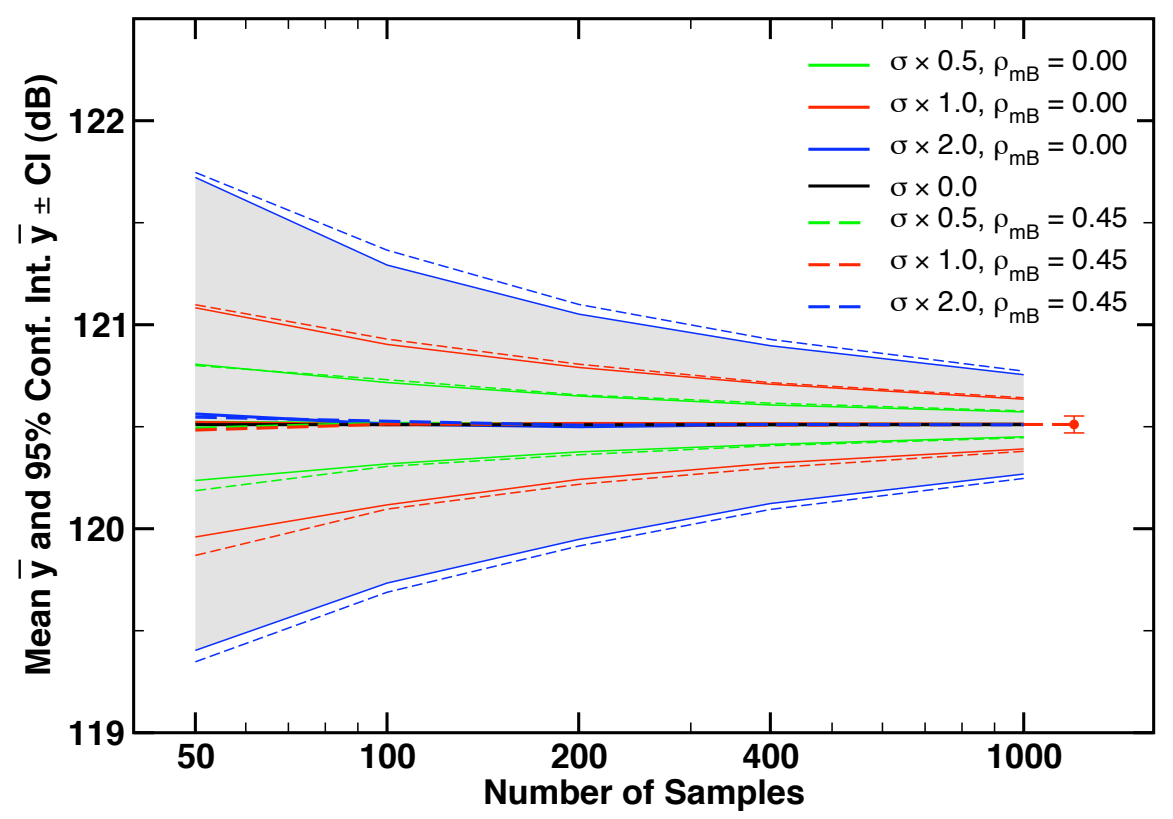

Figure 2: Mean (thick line) and $95 \%$ confidence intervals (CI)(thin line) for sampling equation (3) with normally distributed $m$ and $B$ parameters. $\sigma$ range applies to both parameters where $\sigma_{m}=0.756$ and $\sigma_{B}=1.930$. Solid lines have uncorrelated parameters. Dashed lines have $\rho_{m B}=0.45$. Gray area is region of $95 \%$ CI when $\rho_{m B}=0$. Symbol on right applies to 10000 samples.

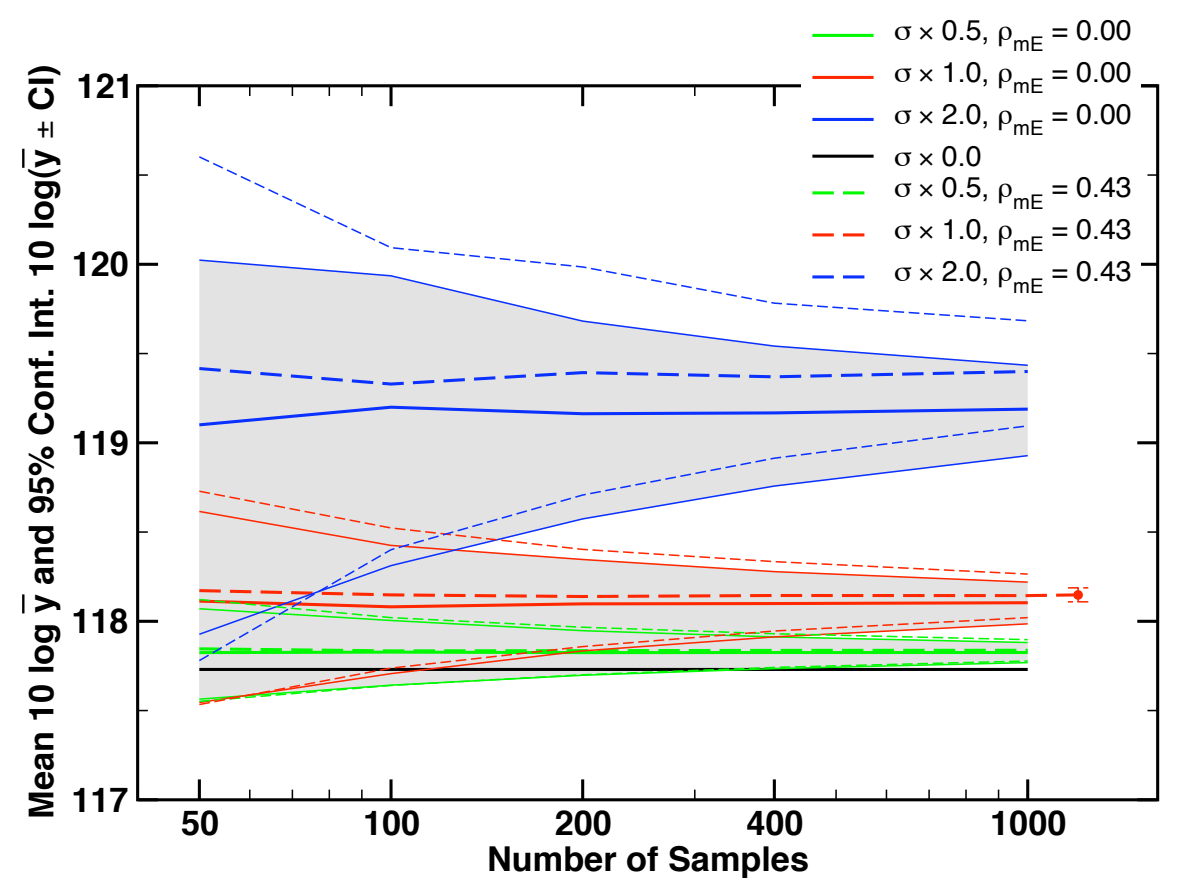

Figure 3: Mean (thick line) and 95\% confidence intervals (CI)(thin line) for sampling equation (6) with normally distributed $m$ and $E$ parameters. $\sigma$ range applies to both parameters where $\sigma_{m}=0.538$ and $\sigma_{E}=0.177$. Solid lines have uncorrelated parameters. Dashed lines have $\rho_{m E}=0.43$. Gray area is region of $95 \%$ CI when $\rho_{m E}=0$. Symbol on right applies to 10000 samples. 


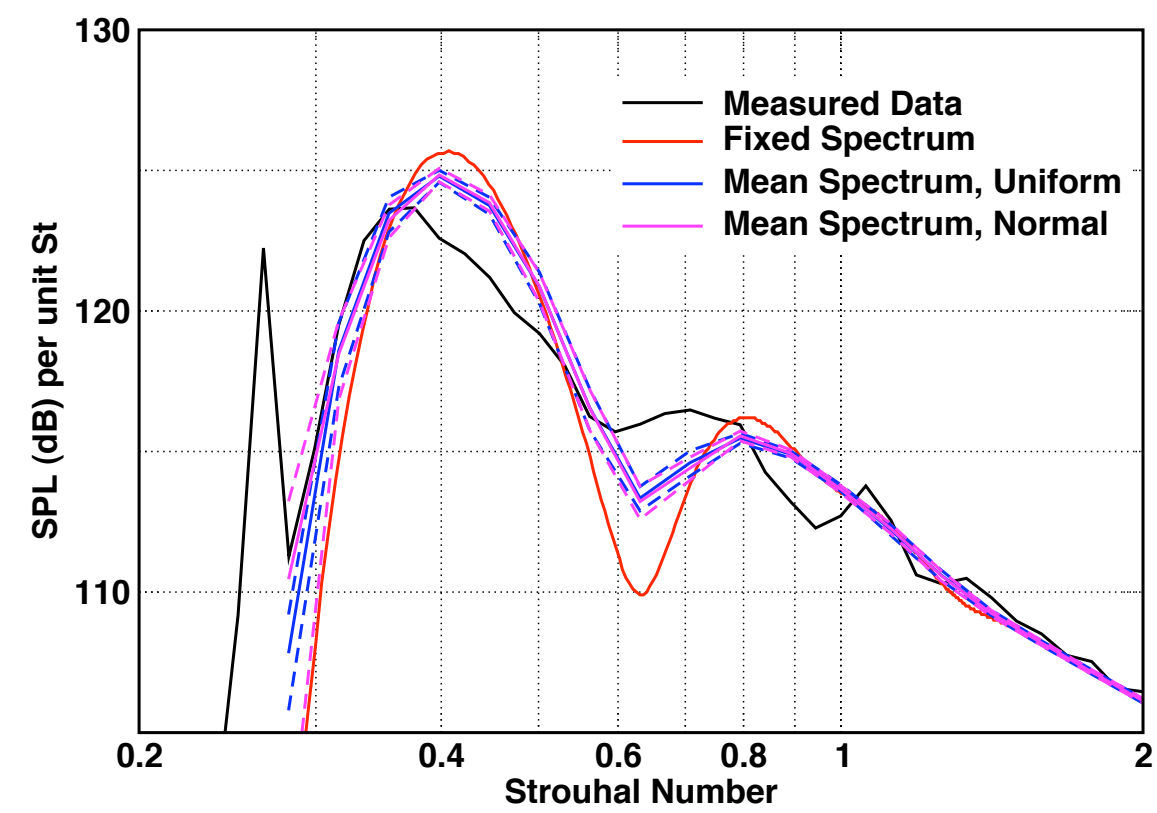

Figure 4: Power spectral density comparisons at 50 degrees, distance $100 D_{e}, M_{j}=1.493, P_{t} / P_{\infty}=3.636$, $T_{t} / T_{\infty}=1.01$. Effect of replacing the $v_{c 1}$ parameter in the Tam model with a uniform distribution $\pm 24 \%$ and a normal distribution both with mean 0.708 and variance $0.00937 . N=100$. Dashed lines for $95 \%$ CI.

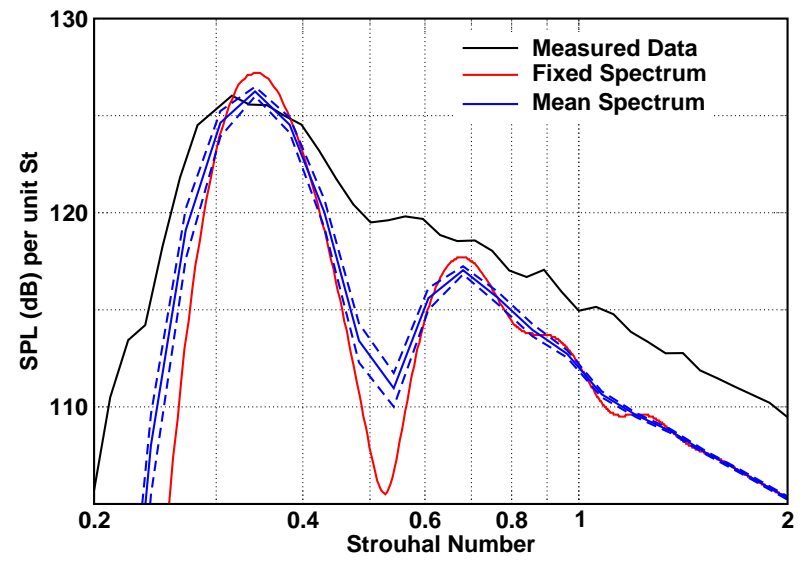

(a) Uniform $\pm 10 \%, N=100$

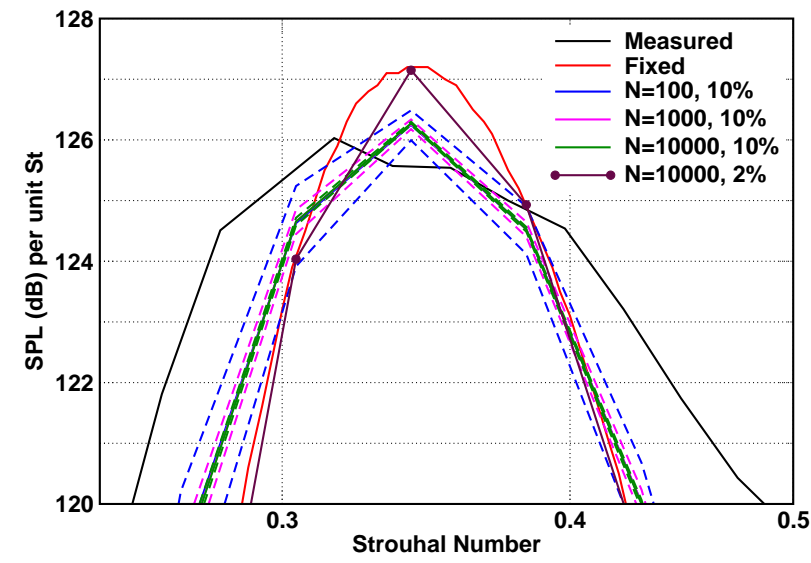

(b) Expanded view, vary $N$ and distribution size

Figure 5: Power spectral density comparisons showing spectra from the Tam model with 12 fixed parameters and spectra from the Tam model with 12 parameters replaced by uniform distributions with $\pm 10 \%$ range with variance 0.00167 or with $\pm 2 \%$ range with variance $6.68 \times 10^{-5}$. Results at 50 degree inlet angle, distance $100 D_{e}, M_{j}=1.494, P_{t} / P_{\infty}=3.629, T_{t} / T_{\infty}=2.16$. 


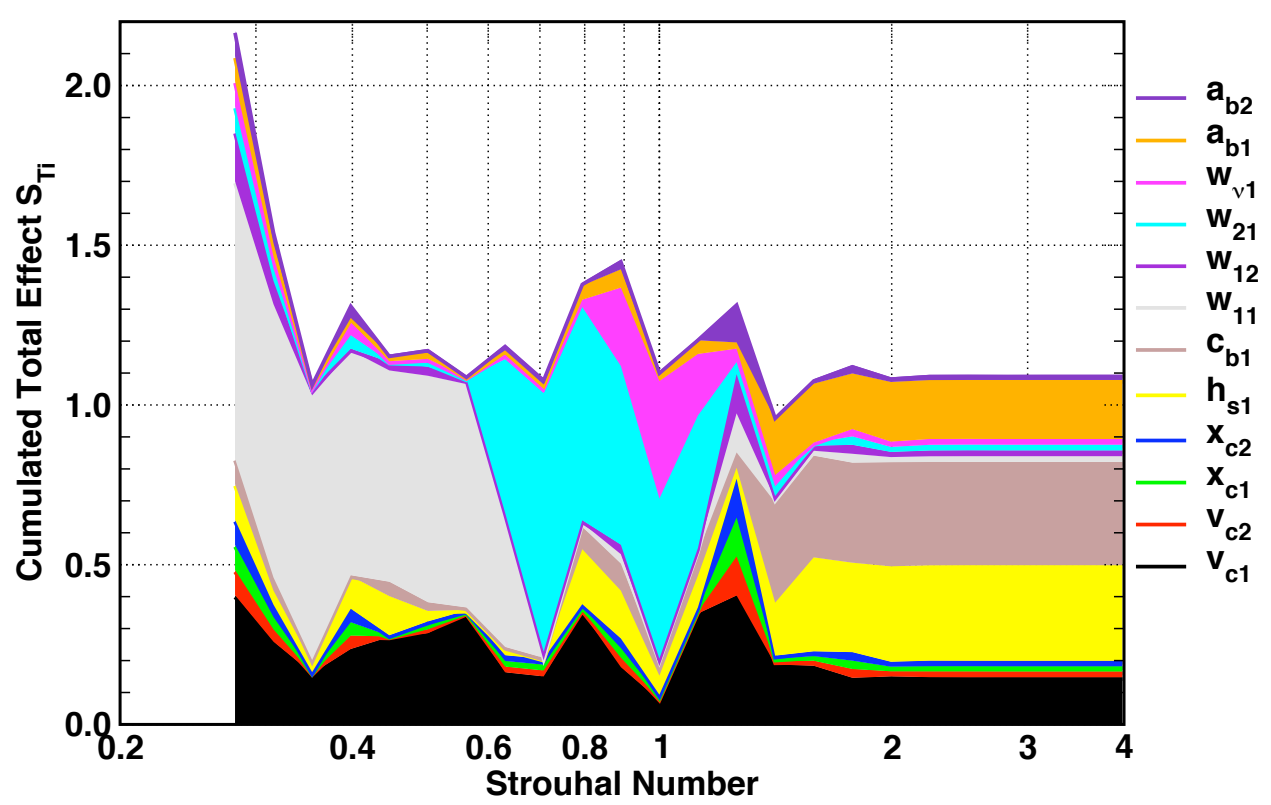

(a) Total effect sensitivity measures derived from variance-based decomposition for the parameters listed in Table 1. Non-influential variables have smaller widths. $N=10000$.

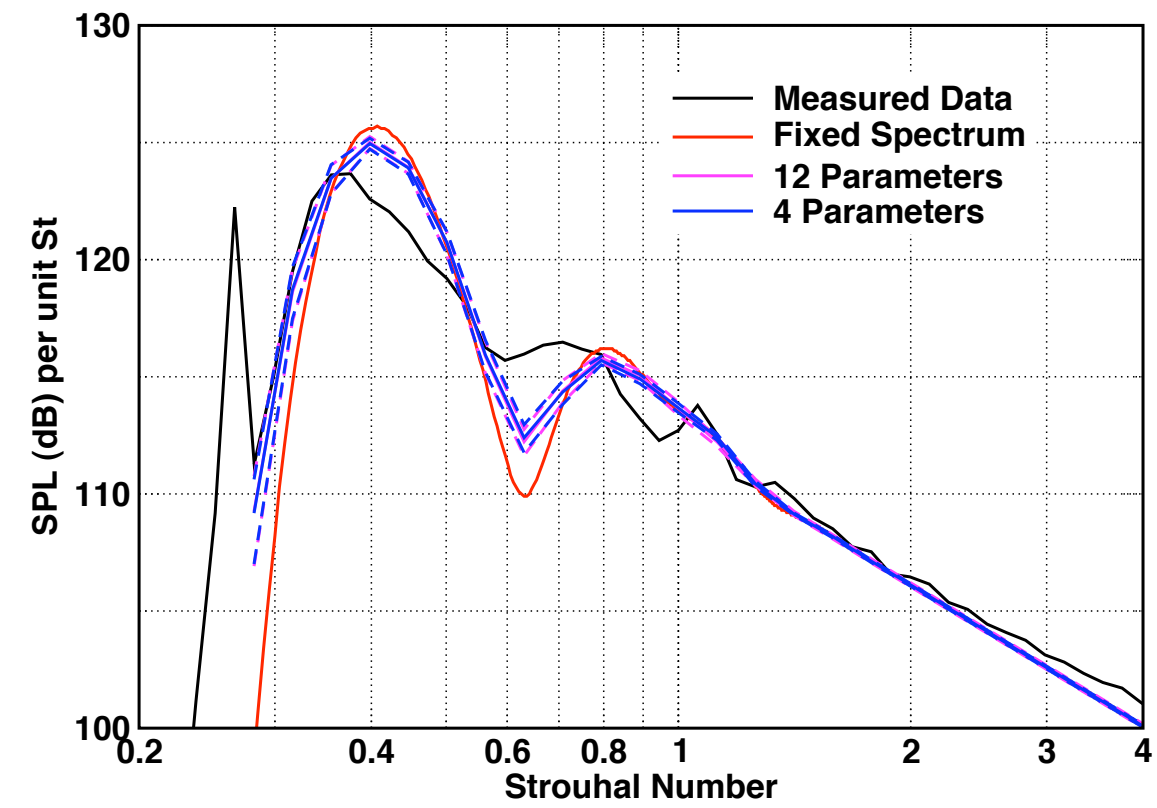

(b) Power spectral density at 50 degrees. Dashed lines for 95\% CI. $N=100$.

Figure 6: Effects of reducing the number of parameters with distributions from 12 to 4 in the Tam model after determining variables to set constant using a variance-based decomposition sensitivity analysis. All parameters set with uniform distributions $\pm 10 \%$. Results at 50 degrees, distance $100 D_{e}, M_{j}=1.493$, $P_{t} / P_{\infty}=3.636, T_{t} / T_{\infty}=1.01$. 


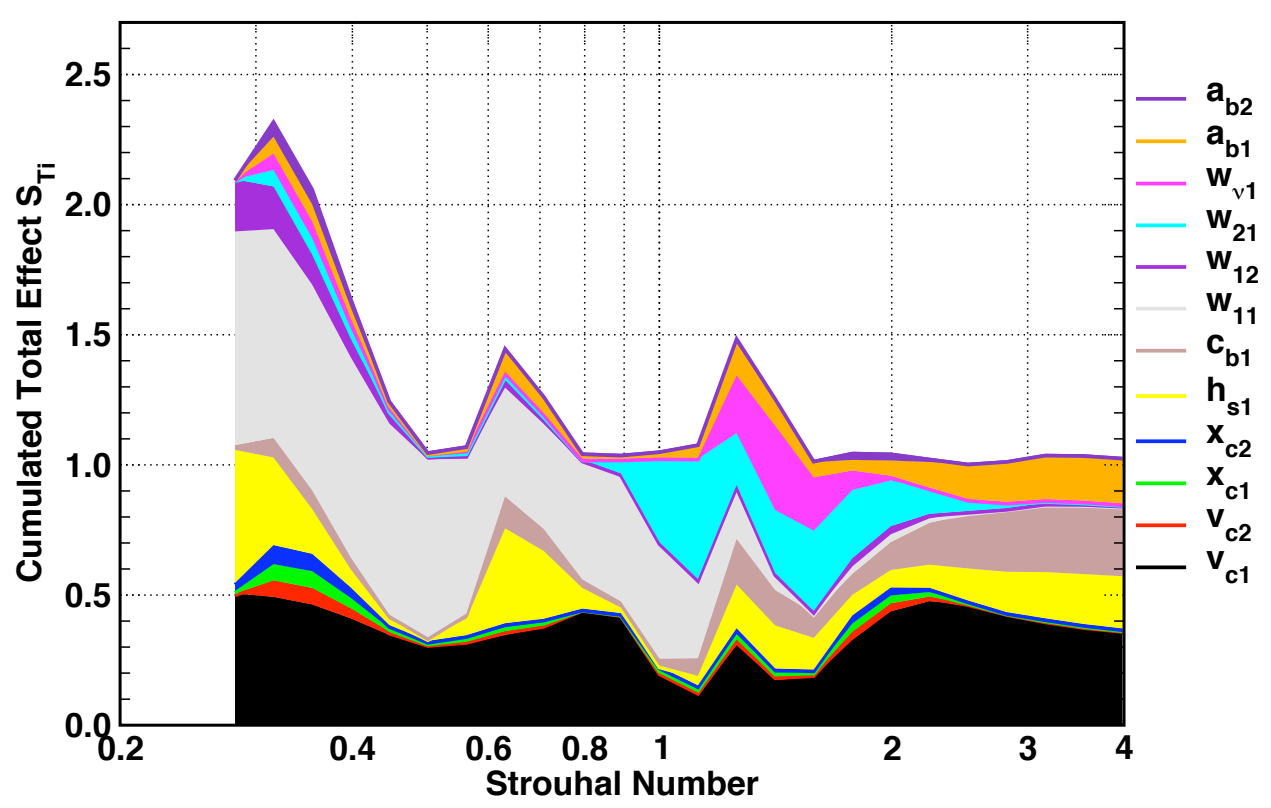

(a) Total effect sensitivity measures derived from variance-based decomposition for the parameters listed in Table 1 . Non-influential variables have smaller widths. $N=10000$.

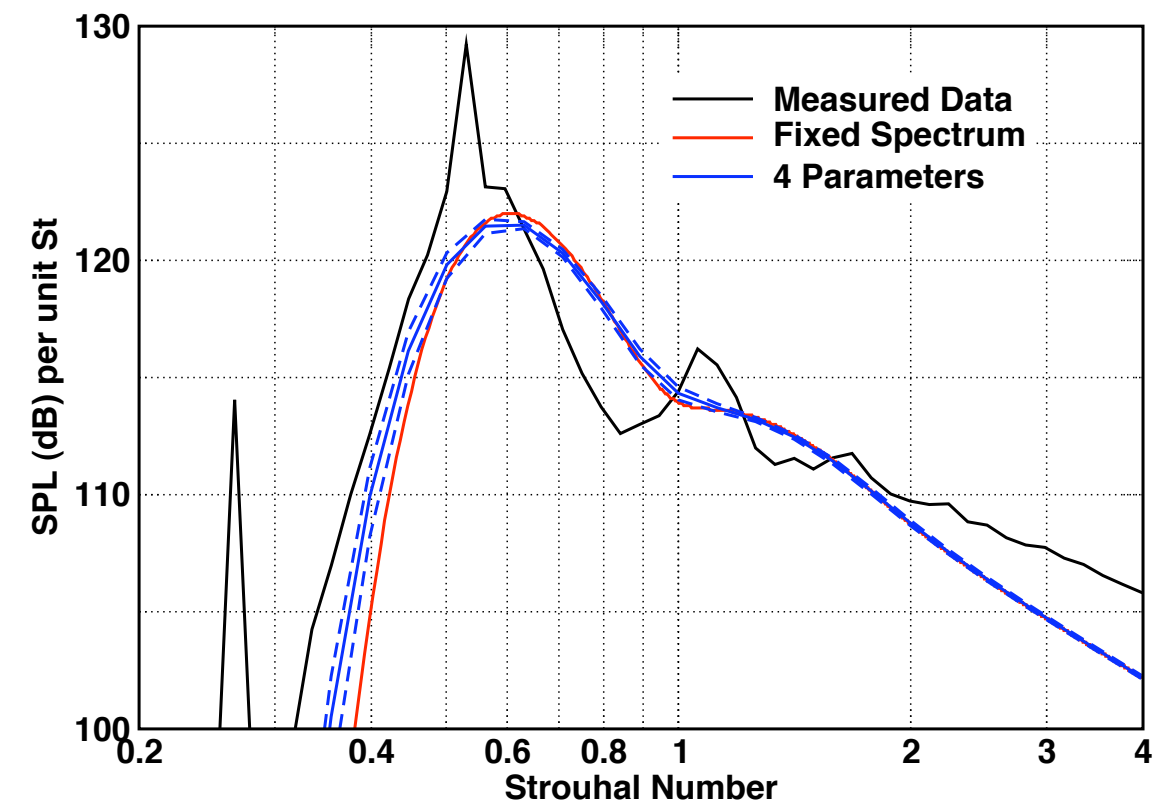

(b) Power spectral density at 90 degrees. Dashed lines for $95 \%$ CI. $N=100$.

Figure 7: Variance-based decomposition sensitivity analysis used to reduce the number of parameters with distributions from 12 to 4 in the Tam model. All parameters set with uniform distributions $\pm 10 \%$. Results at 90 degrees, distance $100 D_{e}, M_{j}=1.493, P_{t} / P_{\infty}=3.636, T_{t} / T_{\infty}=1.01$. 


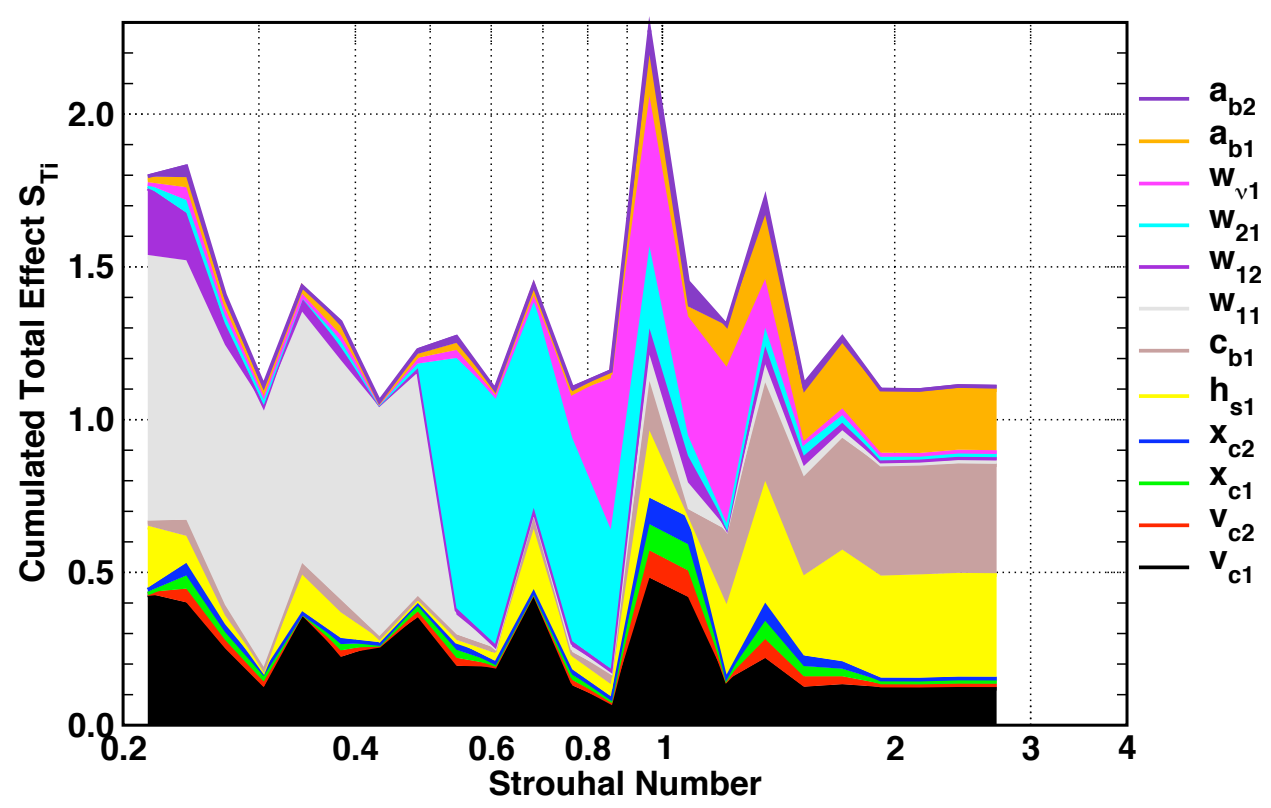

(a) Total effect sensitivity measures derived from variance-based decomposition for the parameters listed in Table 1 . Non-influential variables have smaller widths. $N=10000$.

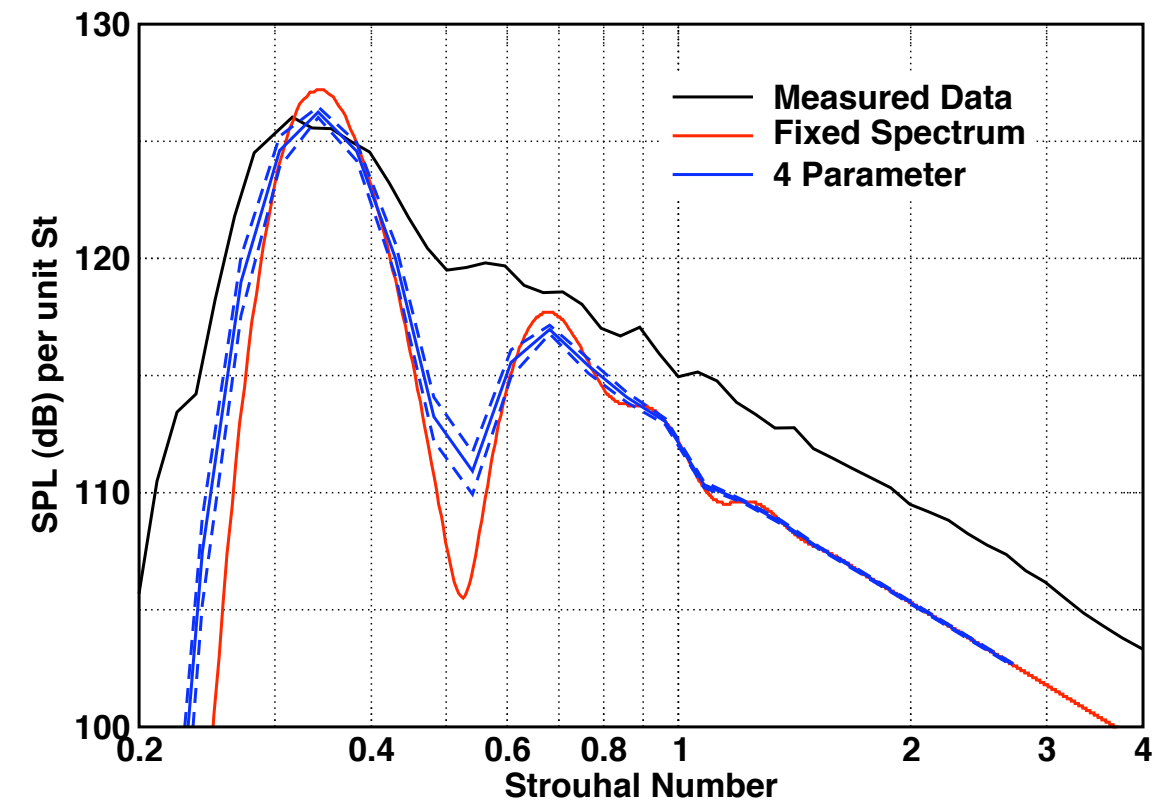

(b) Power spectral density at 50 degrees. Dashed lines for 95\% CI. $N=100$.

Figure 8: Variance-based decomposition sensitivity analysis used to reduce the number of parameters with distributions from 12 to 4 in the Tam model. All parameters set with uniform distributions $\pm 10 \%$. Results at 50 degrees, distance $100 D_{e}, M_{j}=1.494, P_{t} / P_{\infty}=3.629, T_{t} / T_{\infty}=2.16$. 


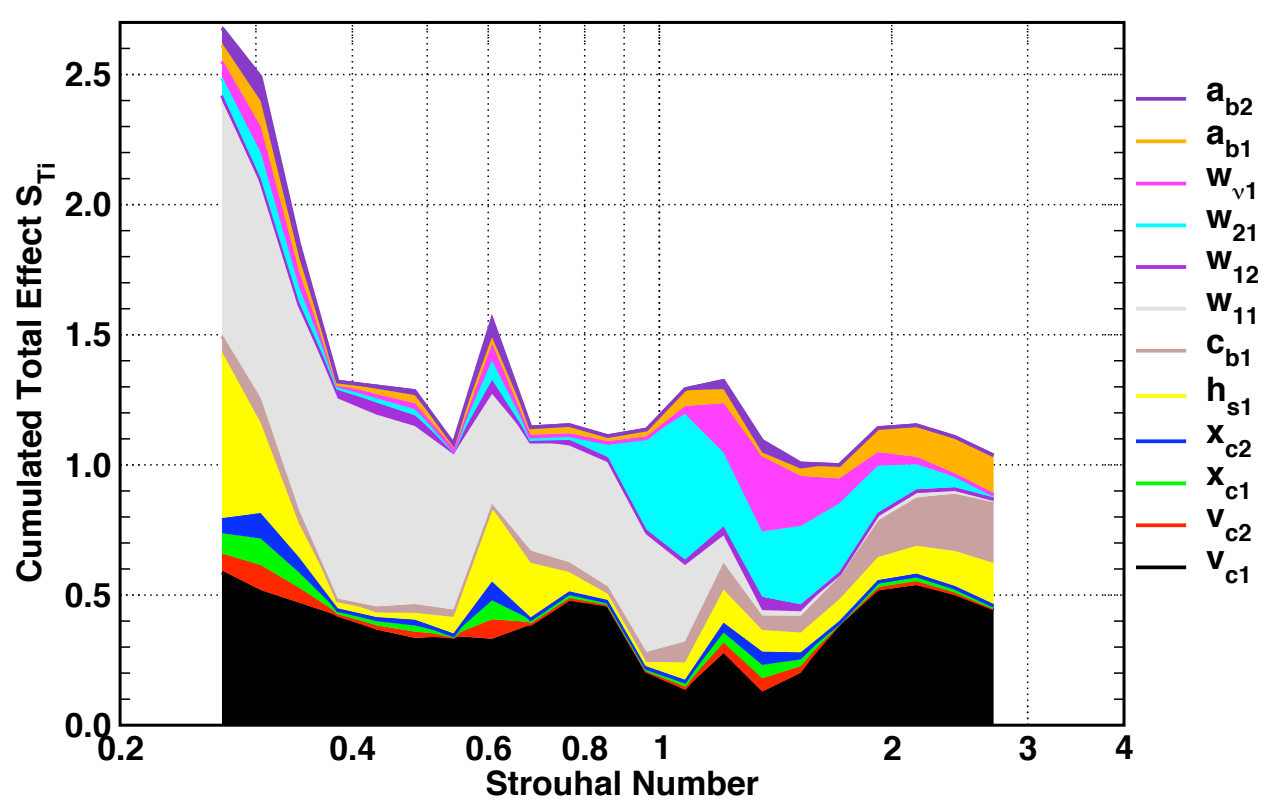

(a) Total effect sensitivity measures derived from variance-based decomposition for the parameters listed in Table 1 . Non-influential variables have smaller widths. $N=10000$.

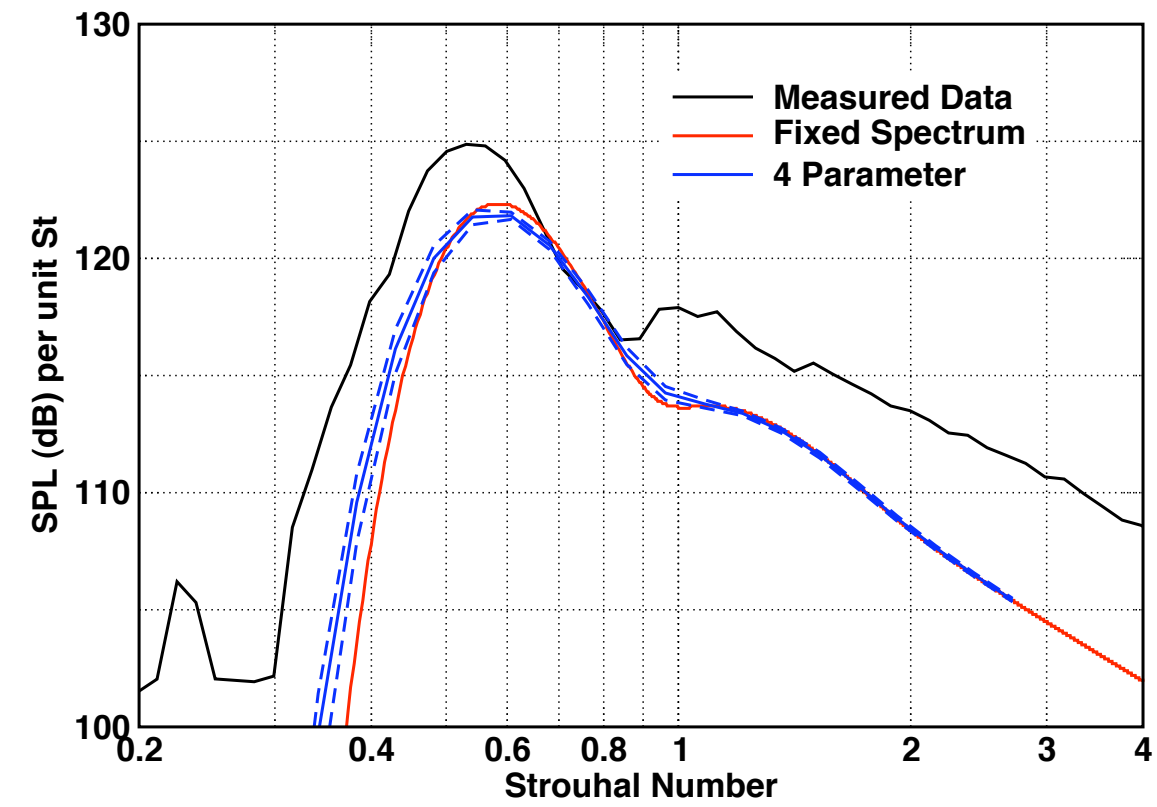

(b) Power spectral density at 90 degrees. Dashed lines for $95 \%$ CI. $N=100$.

Figure 9: Variance-based decomposition sensitivity analysis used to reduce the number of parameters with distributions from 12 to 4 in the Tam model. All parameters set with uniform distributions $\pm 10 \%$. Results at 90 degrees, distance $100 D_{e}, M_{j}=1.494, P_{t} / P_{\infty}=3.629, T_{t} / T_{\infty}=2.16$. 


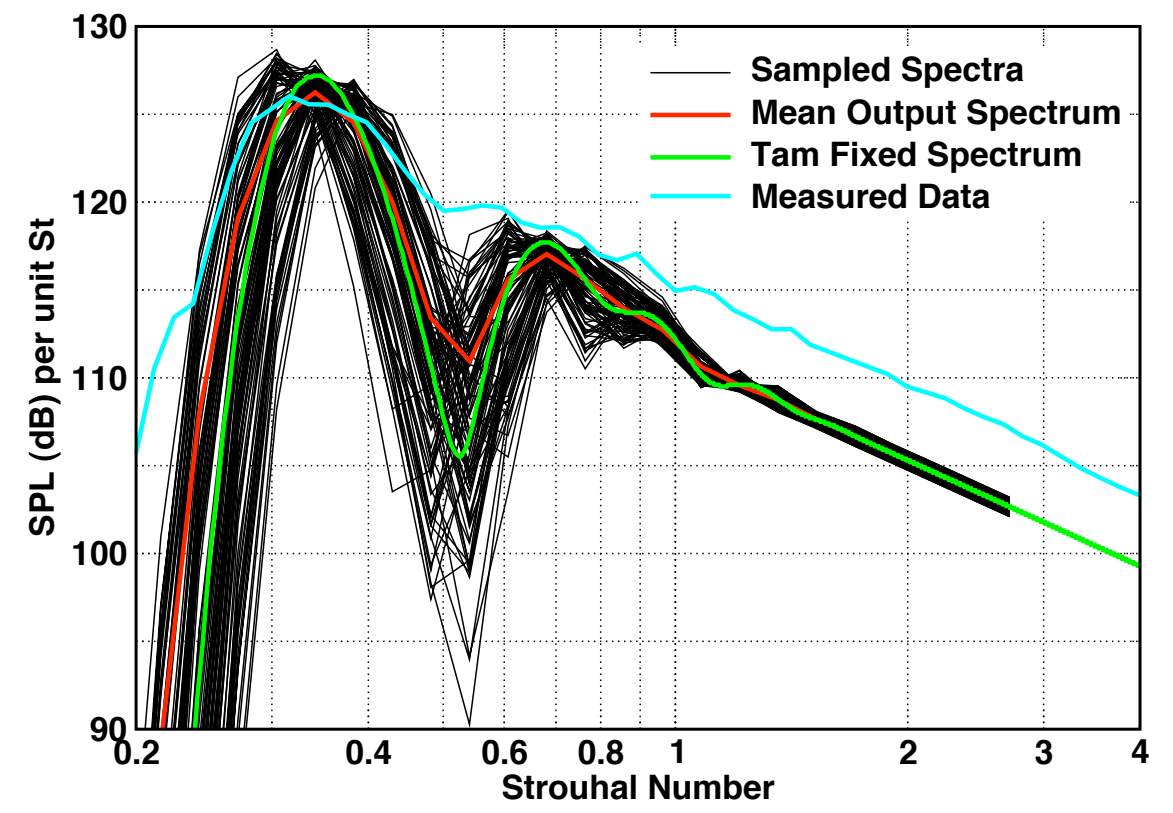

Figure 10: Power spectral density comparisons at 50 degrees, distance $100 D_{e}, M_{j}=1.494, P_{t} / P_{\infty}=$ $3.629, T_{t} / T_{\infty}=2.16$. Scatter of output spectra from the Tam model computed using random samples of 4 parameters with uniform distributions $\pm 10 \%$ with mean output spectrum shown. $N=100$. 


\begin{tabular}{|c|c|c|}
\hline \multicolumn{2}{|c|}{ REPORT DOCUMENTATION PAGE } & $\begin{array}{l}\text { Form Approved } \\
\text { OMB No. 0704-0188 }\end{array}$ \\
\hline \multicolumn{3}{|c|}{ 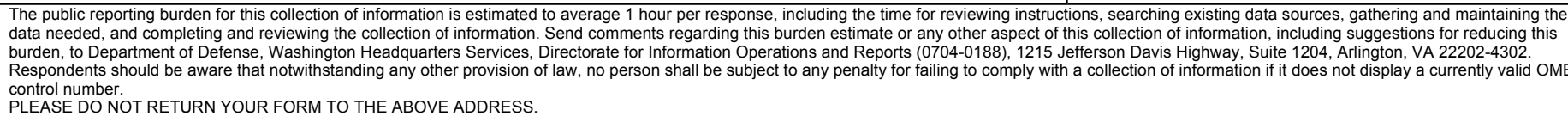 } \\
\hline $\begin{array}{l}\text { 1. REPORT DATE (DD-MM-YYYY) } \\
01-09-2010\end{array}$ & $\begin{array}{l}\text { 2. REPORT TYPE } \\
\text { Technical Memorandum }\end{array}$ & 3. DATES COVERED (From - To) \\
\hline \multirow{3}{*}{\multicolumn{2}{|c|}{$\begin{array}{l}\text { 4. TITLE AND SUBTITLE } \\
\text { The Effect of Nondeterministic Parameters on Shock-Associated N }\end{array}$}} & 5a. CONTRACT NUMBER \\
\hline & & 5b. GRANT NUMBER \\
\hline & & 5c. PROGRAM ELEMENT NUMBER \\
\hline \multirow{3}{*}{\multicolumn{2}{|c|}{$\begin{array}{l}\text { 6. AUTHOR(S) } \\
\text { Dahl, Milo, D.; Khavaran, Abbas }\end{array}$}} & 5d. PROJECT NUMBER \\
\hline & & 5e. TASK NUMBER \\
\hline & & $\begin{array}{l}\text { 5f. WORK UNIT NUMBER } \\
\text { WBS 984754.02.07.03.17.02 }\end{array}$ \\
\hline \multicolumn{2}{|c|}{$\begin{array}{l}\text { 7. PERFORMING ORGANIZATION NAME(S) AND ADDRESS(ES) } \\
\text { National Aeronautics and Space Administration } \\
\text { John H. Glenn Research Center at Lewis Field } \\
\text { Cleveland, Ohio 44135-3191 }\end{array}$} & $\begin{array}{l}\text { 8. PERFORMING ORGANIZATION } \\
\text { REPORT NUMBER } \\
\text { E-17432 }\end{array}$ \\
\hline \multirow{2}{*}{\multicolumn{2}{|c|}{$\begin{array}{l}\text { 9. SPONSORING/MONITORING AGENCY NAME(S) AND ADDRESS(ES) } \\
\text { National Aeronautics and Space Administration } \\
\text { Washington, DC 20546-0001 }\end{array}$}} & $\begin{array}{l}\text { 10. SPONSORING/MONITOR'S } \\
\text { ACRONYM(S) } \\
\text { NASA }\end{array}$ \\
\hline & & $\begin{array}{l}\text { 11. SPONSORING/MONITORING } \\
\text { REPORT NUMBER } \\
\text { NASA/TM-2010-216801 }\end{array}$ \\
\hline \multicolumn{3}{|c|}{$\begin{array}{l}\text { 12. DISTRIBUTION/AVAILABILITY STATEMENT } \\
\text { Unclassified-Unlimited } \\
\text { Subject Category: } 71 \\
\text { Available electronically at http://gltrs.grc.nasa.gov } \\
\text { This publication is available from the NASA Center for AeroSpace Information, 443-757-5802 }\end{array}$} \\
\hline
\end{tabular}

\section{SUPPLEMENTARY NOTES}

\section{ABSTRACT}

Engineering applications for aircraft noise prediction contain models for physical phenomenon that enable solutions to be computed quickly. These models contain parameters that have an uncertainty not accounted for in the solution. To include uncertainty in the solution, nondeterministic computational methods are applied. Using prediction models for supersonic jet broadband shock-associated noise, fixed model parameters are replaced by probability distributions to illustrate one of these methods. The results show the impact of using nondeterministic parameters both on estimating the model output uncertainty and on the model spectral level prediction. In addition, a global sensitivity analysis is used to determine the influence of the model parameters on the output, and to identify the parameters with the least influence on model output.

\section{SUBJECT TERMS}

Jet noise; Noise prediction; Sensitivity analysis; Statistical analysis; Accuracy

\begin{tabular}{|c|c|c|c|c|}
\hline \multicolumn{3}{|c|}{ 16. SECURITY CLASSIFICATION OF: } & \multirow{2}{*}{$\begin{array}{l}\text { 17. LIMITATION OF } \\
\text { ABSTRACT } \\
\text { UU }\end{array}$} & \multirow{2}{*}{$\begin{array}{l}\text { 18. NUMBER } \\
\text { OF } \\
\text { PAGES } \\
30\end{array}$} \\
\hline $\begin{array}{l}\text { a. REPORT } \\
\text { U }\end{array}$ & $\begin{array}{l}\text { b. ABSTRACT } \\
U\end{array}$ & $\begin{array}{l}\text { c. THIS } \\
\text { PAGE } \\
\text { U }\end{array}$ & & \\
\hline
\end{tabular}



